MIT-CTP-2861

OKHEP-99-03

hep-ph/9905329

\title{
Hadron Structure Functions in a Chiral Quark Model: Regularization, Scaling and Sum Rules*
}

\author{
H. Weigel ${ }^{\dagger a}$, E. Ruiz Arriola ${ }^{b}$ and L. Gamberg ${ }^{c}$ \\ ${ }^{a}$ Center for Theoretical Physics \\ Laboratory of Nuclear Science and Department of Physics \\ Massachusetts Institute of Technology \\ Cambridge, Ma 02139 \\ ${ }^{\mathrm{b}}$ Departamento de Física Moderna \\ Universidad de Granada \\ E-18071 Granada, Spain \\ ${ }^{\mathrm{c}}$ Department of Physics and Astronomy \\ University of Oklahoma \\ 440 West Brooks, Norman, Ok 73019
}

\begin{abstract}
We provide a consistent regularization procedure for calculating hadron structure functions in a chiral quark model. The structure functions are extracted from the absorptive part of the forward Compton amplitude in the Bjorken limit. Since this amplitude is obtained as a time-ordered correlation function its regularization is consistently determined from the regularization of the bosonized action. We find that the Pauli-Villars regularization scheme is most suitable because it preserves both the anomaly structure of QCD and the leading scaling behavior of hadron structure functions in the Bjorken limit. We show that this procedure yields the correct pion structure function. In order to render the sum rules of the regularized polarized nucleon structure functions consistent with their corresponding axial charges we find it mandatory to further specify the regularization procedure. This specification goes beyond the double subtraction scheme commonly employed when studying static hadron properties in this model. In particular the present approach serves to determine the regularization prescription for structure functions whose leading moments are not given by matrix elements of local operators. In this regard we conclude somewhat surprisingly that in this model the Gottfried sum rule does not undergo regularization.
\end{abstract}

PACS: 11.30.Rd, 11.55.He, 12.39.-x, 12.39.Fe

Keywords: Structure functions, Compton amplitude, chiral symmetry, Pauli-Villars regularization, soliton model, collective quantization, sum rules

\footnotetext{
* This work is supported in parts by funds provided by the U.S. Department of Energy (D.O.E.) under cooperative research agreements \#DF-FC02-94ER40818, and \#DE-FG03-98ER41066, the Deutsche Forschungsgemeinschaft (DFG) under contract We 1254/3-1 and the Spanish DGES grant no. PB95-1204 and the Junta de Andalucía grant no. FQM0225.

$\dagger_{\text {Heisenberg-Fellow }}$
} 


\section{Introduction}

The analysis of deep inelastic scattering (DIS) provides some of the most convincing evidence for the quark sub-structure [1] of the nucleon because the resulting cross-sections are parameterized by structure functions which exhibit scaling properties characteristic of the quark sub-structure. The parton model parameterizes these structure functions in terms of quark and anti-quark distributions. To leading order in an expansion in twist perturbative QCD in conjunction with the quark-parton model successfully describes the observed violations of the scaling properties. In addition, using the world data the various parameterizations [2] of quark distributions are successfully categorizing a wealth of information about the (quark) sub-structure of hadrons.

However, without bound state wave-functions from first principles in QCD for hadrons it is not possible to calculate the quark distributions and corresponding hadron structure functions. On the other hand, from the point of view of an effective field theory the study of quark momentum distributions in a hadron is particularly challenging, because it requires the coexistence of hadronic and quark degrees of freedom, without perpetration of double counting. As of present there is no obvious expansion parameter which facilitates a rigorous accounting of such contributions. This is to be contrasted with the very low or very high energy regimes where respectively the chiral expansion or perturbative QCD apply. Thus far, one must resort to model calculations [3, 4]. In this context it is important to note that there are the phenomenologically successful chiral models for hadron properties [5]. From the perspective that hadron structure functions can be viewed as functions of quark distributions we adopt the Nambu-Jona-Lasinio (NJL) model [6] of quark flavor dynamics. This model offers a microscopic description of dynamical chiral symmetry breaking for low energy non-perturbative hadron physics. Upon bosonization of the quark fields, meson fields are described as functionals in terms of non-perturbative quark anti-quark bound states [7, 8]. Moreover chiral soliton configurations can be constructed for these meson fields [9]. In this manner the model provides a non-perturbative description not only for the mesons but also the baryons. In the case of the latter the quark fields become functionals of a chiral soliton. During the process of bosonization a link to the quark degrees of freedom is maintained and one is tempted to compare the associated quark distributions to those which enter the quark-parton model description of structure functions. The major reason, however, for adopting this model is that the hadronic tensor, which defines the structure functions, can efficiently be studied in this model. This is based on the observation that prior to bosonization the symmetry currents entering the hadronic tensor are formally identical to currents in a non-interacting Dirac theory. At this formal level the NJL quark distributions can indeed be identified with those of the parton model. However, a major obstacle is that the bosonized NJL model contains quadratic divergences which have to be regularized. This regularization eventually spoils the formal identity of these distributions. For that reason the regularization of pion structure functions has sometimes been imposed $a$ posteriori.

In the NJL chiral soliton model the issue of systematically regularizing the nucleon structure functions has yet to be addressed. Rather various approximations have been made. In 
the first approximation the contributions of the polarized vacuum to the structure functions were omitted [10, 11]. This approach is motivated by the observation that (after regularization) the vacuum contributions to the corresponding static nucleon properties are small once the self-consistent soliton is constructed. Other approaches included vacuum contributions, however, regularization was introduced in a somewhat ad hoc manner by performing a single Pauli-Villars type subtraction [12, 13, 14]. The single subtraction Pauli-Villars treatment is limited to the chirally symmetric case of vanishing current quark masses as this particular case allows one to ignore the quadratically divergent gap equation which is not rendered finite by a single subtraction. In addition, attempts to impose a regularization scheme at the level of quark distributions using error-function and Gaussian damping of the ultraviolet behavior was carried out in ref [13]. In turn the regularization attributed to a given structure function was conjectured to equal that of the corresponding sum rule associated with static nucleon properties. This connection to static properties for setting up the regularization description does not provide a definite answer for structure functions not having a sum rule; i.e., structure functions whose integral cannot be written as a matrix element of a local operator. The major purpose of the present investigation is to establish a consistent regularization procedure for obtaining nucleon structure functions in the NJL model. Also, it happens that the issue of regularization has significant impact for the pion structure functions, thus we will discuss that case as well.

Before bosonization the NJL model is defined in terms of quark fields by the Lagrangian [6]

$$
\mathcal{L}_{\mathrm{NJL}}=\bar{q}\left(i \not \partial-m_{0}\right) q+2 G_{\mathrm{NJL}}\left\{\left(\bar{q} \frac{\vec{\tau}}{2} q\right)^{2}+\left(\bar{q} \frac{\vec{\tau}}{2} i \gamma_{5} q\right)^{2}\right\}
$$

with the quark interaction described by a chirally symmetric quartic potential. The current quark mass, $m_{0}$, parameterizes the small explicit breaking of chiral symmetry. Using functional techniques the quark fields can be integrated out in favor of auxiliary mesonic fields, $\mathcal{M}=$ $S+i P$. According to the chirally symmetric interaction in the Lagrangian (1.1), $S$ and $P$ are scalar and pseudoscalar degrees of freedom, respectively. This results in the bosonized action [7]

$$
\mathcal{A}_{\mathrm{NJL}}=-i N_{C} \operatorname{Tr}_{\Lambda} \log \left\{i \not \partial-m_{0}-\left(S+i \gamma_{5} P\right)\right\}+\frac{1}{4 G} \int d^{4} x \operatorname{tr}\left[\mathcal{M} \mathcal{M}^{\dagger}\right] .
$$

Here the 'cut-off' $\Lambda$ indicates that the quadratically divergent quark loop requires regularization. In order to compute properties of hadrons from the action (1.2) a twofold procedure is in order. First, formal expressions for the symmetry currents have to be extracted. This is straightforwardly accomplished by adding external sources to the Dirac operator and taking the appropriate functional derivatives. Ignoring effects associated with the regularization, the currents would be as simple as $\bar{q} \gamma_{\mu}\left(\gamma_{5}\right) t^{a} q$, with $t^{a}$ being the appropriate flavor generator. Secondly, hadron states are constructed from the effective action which in turn allows one to calculate the relevant matrix elements of the symmetry currents. For the pion this will be a Bethe-Salpeter wave-function which is obtained by expanding the action (1.2) appropriately

\footnotetext{
${ }^{1}$ As the authors carefully noted these regularization schemes were not intended to be self consistent. Similar schemes were applied in 15.
} 
in the fields $S$ and $P$. In the case of the nucleon we will determine a soliton configuration which after collective quantization carries nucleon quantum numbers [16]. These issues will be reviewed in section 2 .

For hadron structure functions we are required to evaluate matrix elements of bi-local current correlation functions which define the hadronic tenor. As is well-known, this tensor parameterizes the strong interaction part of a deep inelastic scattering (DIS) cross-section and is decomposed into form factors. The form factors depend only on the Lorentz invariants $Q^{2}=-q^{2}$ and $x=Q^{2} / 2 p \cdot q$ where $p$ is the hadron momentum and $q$ denotes the momentum transferred to the hadron by the exchange of a virtual gauge boson. In general the hadronic tensor also depends on the spin orientation, $s$, of the hadron as well as the flavor quantum numbers of the hadronic current $J_{\mu}^{a}$ to which the exchanged boson couples,

$$
W_{\mu \nu}^{a b}(p, q ; s)=\frac{1}{4 \pi} \int d^{4} x e^{i q \cdot \xi}\left\langle p, s\left|\left[J_{\mu}^{a}(\xi), J_{\nu}^{b \dagger}(0)\right]\right| p, s\right\rangle
$$

As the currents are rigorously determined within the model the hadronic tensor is a welldefined quantity and except for calculational matters no further assumptions are needed to compute $W_{\mu \nu}$.

In the case of the nucleon, the form factors appear in the Lorentz-covariant decomposition (omitting parity violating contributions)

$$
\begin{aligned}
W_{\mu \nu}^{a b}(p, q ; s)= & \left(-g_{\mu \nu}+\frac{q_{\mu} q_{\nu}}{q^{2}}\right) M_{N} W_{1}\left(x, Q^{2}\right) \\
& +\left(p_{\mu}-q_{\mu} \frac{p \cdot q}{q^{2}}\right)\left(p_{\nu}-q_{\nu} \frac{p \cdot q}{q^{2}}\right) \frac{1}{M_{N}} W_{2}\left(x, Q^{2}\right) \\
& +i \epsilon_{\mu \nu \lambda \sigma} \frac{q^{\lambda} M_{N}}{p \cdot q}\left(\left[g_{1}\left(x, Q^{2}\right)+g_{2}\left(x, Q^{2}\right)\right] s^{\sigma}-\frac{q \cdot s}{q \cdot p} p^{\sigma} g_{2}\left(x, Q^{2}\right)\right) .
\end{aligned}
$$

When a spin-zero hadron is considered, as e.g. the pion, the polarized structure functions $g_{1}$ and $g_{2}$ are ignored, $c f$. eq (3.1). Once the hadronic tensor is computed, the form factors are given by suitable projections. Finally the leading twist contributions to the structure functions are obtained from these form factors by assuming the Bjorken limit:

$$
Q^{2} \rightarrow \infty \quad \text { with } \quad x=Q^{2} / 2 p \cdot q \text { fixed }
$$

For the spin independent part the structure functions $f_{i}$ are the linear combinations

$$
M_{N} W_{1}\left(x, Q^{2}\right) \stackrel{\mathrm{Bj}}{\longrightarrow} f_{1}(x) \text { and } \frac{p \cdot q}{M_{N}} W_{2}\left(x, Q^{2}\right) \stackrel{\mathrm{Bj}}{\longrightarrow} f_{2}(x) .
$$

However, the expression (1.3) is not conveniently computed from the bosonized action (1.2). In case the hadron represents the ground state with the specific quantum numbers, the hadronic tensor will be related to the forward virtual Compton amplitude

$$
W_{\mu \nu}^{a b}(p, q ; s)=\frac{1}{2 \pi} \operatorname{Im} T_{\mu \nu}^{a b}(p, q ; s),
$$


which is given as the matrix element of a time-ordered product of the currents

$$
T_{\mu \nu}^{a b}(p, q ; s)=i \int d^{4} \xi e^{i q \cdot \xi}\left\langle p, s\left|T\left\{J_{\mu}^{a}(\xi) J_{\nu}^{b \dagger}(0)\right\}\right| p, s\right\rangle
$$

rather than their commutator. This time-ordered product can easily be obtained by expanding the bosonized action (1.2) up to quadratic order in the external fields. Applying Cutkosky's rules subsequently yields the absorptive part, $\operatorname{Im} T_{\mu \nu}$, which is to be identified with the hadronic tensor (1.7).

The paper is organized as follows. In section 2 we will review the model with regard to the meson and soliton sectors. In section 3 we will discuss in detail the calculation of the pion structure function. In the fully regularized model this goes beyond simply computing the standard 'handbag' diagram. Fortunately the Bjorken limit provides some simplifications. In section 4 will will discuss how these simplifications can be transferred to the soliton sector; thereby exhibiting the leading scaling laws. Section 5 represents the main part of the paper providing formal expressions for the nucleon structure functions in the fully regularized NJL chiral soliton model. In section 6 we will perform consistency checks with regard to the pertinent sum rules. We will summarize our results in section 7. Starting from the Bethe-Salpeter amplitude an alternative computation of the pion structure function is presented in appendix A while appendix $\mathrm{B}$ contains a formal discussion on the momentum distribution among the propagators in the quark loop. In appendix $\mathrm{C}$ we will present a brief discussion of the (unphysical) model which has been included to regularize the quadratically divergent action (1.2). Finally appendix D contains some lengthy expressions for the nucleon structure functions at sub-leading order in $1 / N_{C}$. At this order the structure functions which enter the Alder and Gottfried sum rules are non-vanishing. Surprisingly we find that the latter does not undergo regularization. Some preliminary results of the present studies have already been communicated previously [17, 18].

\section{Pauli-Villars regularization for the NJL model}

Upon employing the Pauli-Villars regularization scheme it is possible to formulate the bosonized NJL model [6] completely in Minkowski space. This will be quite appropriate when applying Cutkosky's rules in order to extract the hadronic tensor from the Compton amplitude. Also, let us remind the reader that in ref [17], scaling for the pion structure functions was accomplished in the Pauli-Villars regularization, and not in the proper-time regularization. In this context, it has been shown [19] that in the Pauli-Villars regularization, unlike the more customary proper-time scheme where cuts in the complex plane appear [20, 9], dispersion relations are fulfilled.

\section{2a. Vacuum and meson sectors}

In this subsection we will set the stage for our investigation of structure functions by first reviewing the vacuum and meson sectors of the NJL model. We will be as brief as possible because these issues have been thoroughly discussed in the literature [7, 8, 9]. The Pauli-Villars regularization has been considered before in this context both for mesons and solitons and we

refer to refs [17, 19, 21, 22, 23, 24] for more details and results in this regularization scheme. 
We will specifically follow ref [17] because in that formulation a consistent treatment solely in Minkowski space is possible.

We consider two different Dirac operators in the background of scalar $(S)$ and pseudoscalar $(P)$ fields 17, 21]

$$
\begin{aligned}
i \mathbf{D} & =i \not \partial-\left(S+i \gamma_{5} P\right)+\psi+\not h \gamma_{5}=: i \mathbf{D}^{(\pi)}+\psi+\not h \gamma_{5} \\
i \mathbf{D}_{5} & =-i \not \partial-\left(S-i \gamma_{5} P\right)-\psi+\not h \gamma_{5}=: i \mathbf{D}_{5}^{(\pi)}-\psi+\not h \gamma_{5} .
\end{aligned}
$$

Here we have also introduced external vector $\left(v_{\mu}\right)$ and axial-vector $\left(a_{\mu}\right)$ fields. As noted above the functional derivate of the action with respect to these sources will provide the vector and axial-vector currents, respectively. For later use we have also defined Dirac operators, $\mathbf{D}^{(\pi)}$ and $\mathbf{D}_{5}^{(\pi)}$, with these fields omitted. Of course, all fields appearing in eqs (2.1) and (2.2) are considered to be matrix fields in flavor space. It is worth noting that upon continuation to Euclidean space, $\mathbf{D}_{5}$ transforms into the hermitian conjugate of $\mathbf{D}$ [21.

The regularized action of the bosonized NJL model is then given by

$$
\begin{aligned}
\mathcal{A}_{\mathrm{NJL}} & =\mathcal{A}_{\mathrm{R}}+\mathcal{A}_{\mathrm{I}}+\frac{1}{4 G} \int d^{4} x \operatorname{tr}\left[S^{2}+P^{2}+2 m_{0} S\right] \\
\mathcal{A}_{\mathrm{R}} & =-i \frac{N_{C}}{2} \sum_{i=0}^{2} c_{i} \operatorname{Tr} \log \left[-\mathbf{D D}_{5}+\Lambda_{i}^{2}-i \epsilon\right] \\
\mathcal{A}_{\mathrm{I}} & =-i \frac{N_{C}}{2} \operatorname{Tr} \log \left[-\mathbf{D}\left(\mathbf{D}_{5}\right)^{-1}-i \epsilon\right] .
\end{aligned}
$$

The local term in eq (2.3) is the remainder of the quartic quark interaction of the NJL model. After having shifted the meson fields by an amount proportional to the current quark masses $m_{0}$ it also contains the explicit breaking of chiral symmetry. Furthermore we have retained the notion of real and imaginary parts of the action as it would come about in the Euclidean space formulation. This is also indicated by the Feynman boundary conditions. When disentangling these pieces, it is found that only the 'real part' $\mathcal{A}_{\mathrm{R}}$ is ultraviolet divergent. It is regularized within the Pauli-Villars scheme according to which the conditions?

$$
c_{0}=1, \quad \Lambda_{0}=0, \quad \sum_{i=0}^{2} c_{i}=0 \quad \text { and } \quad \sum_{i=0}^{2} c_{i} \Lambda_{i}^{2}=0
$$

hold. The 'imaginary part' $\mathcal{A}_{\mathrm{I}}$ is conditionally convergent, i.e. a principle value description must be imposed for the integration over the time coordinate. A priori this does not imply that it should not be regularized. However, in order to correctly reproduce the axial anomaly we are constrained to leave it unregularized [25, 7]. In the case of vector interaction the situation is a bit more involved because suitable counterterms have to be added [21, 26].

Essentially we have added and subtracted the (unphysical) $\mathbf{D}_{5}$ model to the bosonized NJL model. Under regularization the sum, $\log (\mathbf{D})+\log \left(\mathbf{D}_{5}\right)$ is then treated differently from

\footnotetext{
${ }^{1}$ Here and henceforth we denote traces of discrete indices by "tr" while "Tr" also contains the space-time integration.

${ }^{2}$ In the case of two subtractions we need at least two cut-offs $\Lambda_{1}$ and $\Lambda_{2}$. In the limit $\Lambda_{1} \rightarrow \Lambda_{2}=\Lambda$, we have $\sum_{i} c_{i} f\left(\Lambda_{i}^{2}\right)=f(0)-f\left(\Lambda^{2}\right)+\Lambda^{2} f^{\prime}\left(\Lambda^{2}\right)$. For instance, $\sum_{i} c_{i} \Lambda_{i}^{2 n}=(2 n-2) \Lambda^{2 n}$.
} 
the difference, $\log (\mathbf{D})-\log \left(\mathbf{D}_{5}\right)$. In the case of the polarized nucleon structure functions we will later recognize that this special choice of regularization nevertheless requires further specification.

In the vacuum sector the pseudoscalar fields vanish while the variation of the action with respect to the scalar field $S$ and yields the gap equation

$$
\frac{1}{2 G}\left(m-m_{0}\right)=-4 i N_{C} m \sum_{i=0}^{2} c_{i} \int \frac{d^{4} k}{(2 \pi)^{4}}\left[-k^{2}+m^{2}+\Lambda_{i}^{2}-i \epsilon\right]^{-1} .
$$

This equation determines the vacuum expectation value of the scalar field $\langle S\rangle=m$ which is referred to as the constituent quark mass. Its non-vanishing value signals the dynamical breaking of chiral symmetry. Next we expand the action to quadratic order in the pion field $\vec{\pi}$. This field resides in the non-linear representation of the meson fields on the chiral circle

$$
\mathcal{M}=m U=m \exp \left(i \frac{g}{m} \vec{\pi} \cdot \vec{\tau}\right) .
$$

This representation also defines the chiral field $U$. The quark-pion coupling $g$ will be specified shortly. Upon Fourier transforming to $\overrightarrow{\tilde{\pi}}$ we find

$$
\mathcal{A}_{\mathrm{NJL}}=g^{2} \int \frac{d^{4} q}{(2 \pi)^{4}} \overrightarrow{\tilde{\pi}}(q) \cdot \overrightarrow{\tilde{\pi}}(-q)\left[2 N_{C} q^{2} \Pi\left(q^{2}\right)-\frac{1}{2 G} \frac{m_{0}}{m}\right]+\mathcal{O}\left(\vec{\pi}^{4}\right)
$$

with the polarization function

$$
\begin{aligned}
\Pi\left(q^{2}, x\right) & =-i \sum_{i=0}^{2} c_{i} \frac{d^{4} k}{(2 \pi)^{4}}\left[-k^{2}-x(1-x) q^{2}+m^{2}+\Lambda_{i}^{2}-i \epsilon\right]^{-2} \text { and } \\
\Pi\left(q^{2}\right) & =\int_{0}^{1} d x \Pi\left(q^{2}, x\right),
\end{aligned}
$$

parameterizing the quark loop. The on-shell condition for the pion relates its mass to the model parameters

$$
m_{\pi}^{2}=\frac{1}{2 G} \frac{m_{0}}{m} \frac{1}{2 N_{C} \Pi\left(m_{\pi}^{2}\right)} .
$$

Requiring a unit residuum at the pion pole determines the quark-pion coupling

$$
\frac{1}{g^{2}}=\left.4 N_{C} \frac{d}{d q^{2}}\left[q^{2} \Pi\left(q^{2}\right)\right]\right|_{q^{2}=m_{\pi}^{2}} .
$$

The axial current is obtained from the linear coupling to the axial-vector source $a_{\mu}$. Its matrix element between the vacuum and the properly normalized one-pion state provides the pion decay constant $f_{\pi}$ as a function of the model parameters

$$
f_{\pi}=4 N_{C} m g \Pi\left(m_{\pi}^{2}\right) .
$$

The empirical values $m_{\pi}=138 \mathrm{MeV}$ and $f_{\pi}=93 \mathrm{MeV}$ are used to determine the model parameters. 


\section{2b. Soliton sector}

In order to describe a soliton configuration we consider static meson configurations. In that case it is suitable to introduce a Dirac Hamiltonian $h$ via

$$
i \mathbf{D}^{(\pi)}=\beta\left(i \partial_{t}-h\right) \quad \text { and } \quad i \mathbf{D}_{5}^{(\pi)}=\left(-i \partial_{t}-h\right) \beta .
$$

For a given meson configuration the Hamiltonian $h$ is diagonalized

$$
h \Psi_{\alpha}=\epsilon_{\alpha} \Psi_{\alpha},
$$

yielding eigen-spinors $\Psi_{\alpha}$ and energy eigenvalues $\epsilon_{\alpha}$. In the unit baryon number sector the wellknown hedgehog configuration minimizes the action for the meson fields. This configuration introduces the chiral angle $\Theta(r)$ via

$$
h=\vec{\alpha} \cdot \vec{p}+\beta m \exp \left[i \overrightarrow{\hat{r}} \cdot \vec{\tau} \gamma_{5} \Theta(r)\right] .
$$

The eigenstates $|\alpha\rangle$ of this Dirac Hamiltonian are in particular characterized by their grand-spin quantum number [27]. The grand-spin, $\vec{G}$ is the operator sum of total spin and isospin. Since $\vec{G}$ commutes with the Dirac Hamiltonian (2.16) the state $\exp \left(i \pi G_{2}\right)|\alpha\rangle$ is also an eigenstate of (2.16) with energy $\epsilon_{\alpha}$ and grand-spin $G_{\alpha}$. This rotational symmetry, which actually is a grandspin reflection, will later be useful to simplify matrix elements of the quark wave-functions, $\Psi_{\alpha}$.

For unit baryon number configurations it turns out that one distinct level, $\Psi_{\text {val }}$, is strongly bound [9]. This level is referred to as the valence quark state. The total energy functional contains three pieces. The first one is due to the explicit occupation of the valence quark level to ensure unit baryon number. The second is the contribution of the polarized vacuum. It is extracted from the action (2.3) by considering an infinite time interval to discretize the eigenvalues of $\partial_{t}$. The sum over these eigenvalues then becomes a spectral integral [28] which can be computed using Cauchy's theorem. Finally, there is the trivial part stemming from the local part of the action (1.2). Collecting these pieces we have [29, 9]

$$
\begin{gathered}
E_{\text {tot }}[\Theta]=\frac{N_{C}}{2}\left(1-\operatorname{sign}\left(\epsilon_{\text {val }}\right)\right) \epsilon_{\text {val }}-\frac{N_{C}}{2} \sum_{i=0}^{2} c_{i} \sum_{\alpha}\left\{\sqrt{\epsilon_{\alpha}^{2}+\Lambda_{i}^{2}}-\sqrt{\epsilon_{\alpha}^{(0) 2}+\Lambda_{i}^{2}}\right\} \\
+m_{\pi}^{2} f_{\pi}^{2} \int d^{3} r(1-\cos (\Theta)) .
\end{gathered}
$$

Here we have also subtracted the vacuum energy associated with the trivial meson field configuration and made use of the expressions obtained for $m_{\pi}$ and $f_{\pi}$ in the preceding subsection. The soliton is then obtained as the profile function $\Theta(r)$ which minimizes the total energy $E_{\text {tot }}$ self-consistently.

At this point we have constructed a state which has unit baryon number but neither good quantum numbers for spin and flavor. Such states are generated by canonically quantizing the time-dependent collective coordinates $A(t)$ which parameterize the spin-flavor orientation of the soliton. For a rigidly rotating soliton the Dirac operator becomes, after transforming to the 
flavor rotating frame[ [28],

$$
i \mathbf{D}^{(\pi)}=A \beta\left(i \partial_{t}-\vec{\tau} \cdot \vec{\Omega}-h\right) A^{\dagger} \quad \text { and } \quad i \mathbf{D}_{5}^{(\pi)}=A\left(-i \partial_{t}+\vec{\tau} \cdot \vec{\Omega}-h\right) \beta A^{\dagger} .
$$

Actual computations involve an expansion with respect to the angular velocities

$$
A^{\dagger} \frac{d}{d t} A=\frac{i}{2} \vec{\tau} \cdot \vec{\Omega}
$$

According to the quantization rules, the angular velocities are replaced by the spin operator

$$
\vec{\Omega} \longrightarrow \frac{1}{\alpha^{2}} \vec{J}
$$

The constant of proportionality is the moment of inertia $\alpha^{2}$ which is calculated as a functional of the soliton [28]. For the present purpose we remark that $\alpha^{2}$ is of the order $1 / N_{C}$. Hence an expansion in $\vec{\Omega}$ is equivalent to one in $1 / N_{C}$. The nucleon wave-function becomes a (Wigner D) function of the collective coordinates. A useful relation in computing matrix elements of nucleon states is [16]

$$
\left\langle N\left|\frac{1}{2} \operatorname{tr}\left(A^{\dagger} \tau_{i} A \tau_{j}\right)\right| N\right\rangle=-\frac{4}{3}\left\langle N\left|I_{i} J_{j}\right| N\right\rangle
$$

\section{A reminder on the pion structure function}

Here we will reconsider the computation of the pion structure function [17]. The reason is that in the present formulation of the model this calculation goes beyond considering the standard 'handbag' diagram because dimension five operators are involved. The matrix elements of these operators will lead to isospin breaking contributions. The particular way these contributions cancel among each other will teach us how to organize the soliton calculation efficiently.

DIS off pions is characterized by a single structure function, $F(x)$,

$$
\frac{1}{2 \pi} \operatorname{Im} T_{\mu \nu}(p, q) \stackrel{\mathrm{Bj}}{\longrightarrow} F(x)\left[-g_{\mu \nu}+\frac{q_{\mu} q_{\nu}}{q^{2}}-\frac{1}{q^{2}}\left(p_{\mu}-\frac{q_{\mu}}{2 x}\right)\left(p_{\nu}-\frac{q_{\nu}}{2 x}\right)\right],
$$

where the Bjorken limit (1.5) has been indicated. In order to compute the Compton amplitude (3.1) we calculate the time- ordered product

$$
T\left\{J_{\mu}(\xi) J_{\nu}(0)\right\}=\left.\frac{\delta^{2}}{\delta v^{\mu}(\xi) \delta v^{\nu}(0)} \mathcal{A}_{\mathrm{NJL}}[\psi \mathcal{Q}]\right|_{v_{\mu}=0}
$$

\footnotetext{
${ }^{3}$ A generalization to three flavors proceeds analogously but also requires to include flavor symmetry breaking 30 .

${ }^{4}$ When formally considering nucleon structure functions it will turn out that it is mostly sufficient to refer to the Dirac operators as defined in eqs (2.18) rather than to explicitly carry out the expansion in the angular velocities.
} 
Figure 3.1: Local diagrams which do not contribute to the absorptive part of the Compton amplitude in the Bjorken limit. Dotted and curly lines refer to the pion and photon fields, respectively.

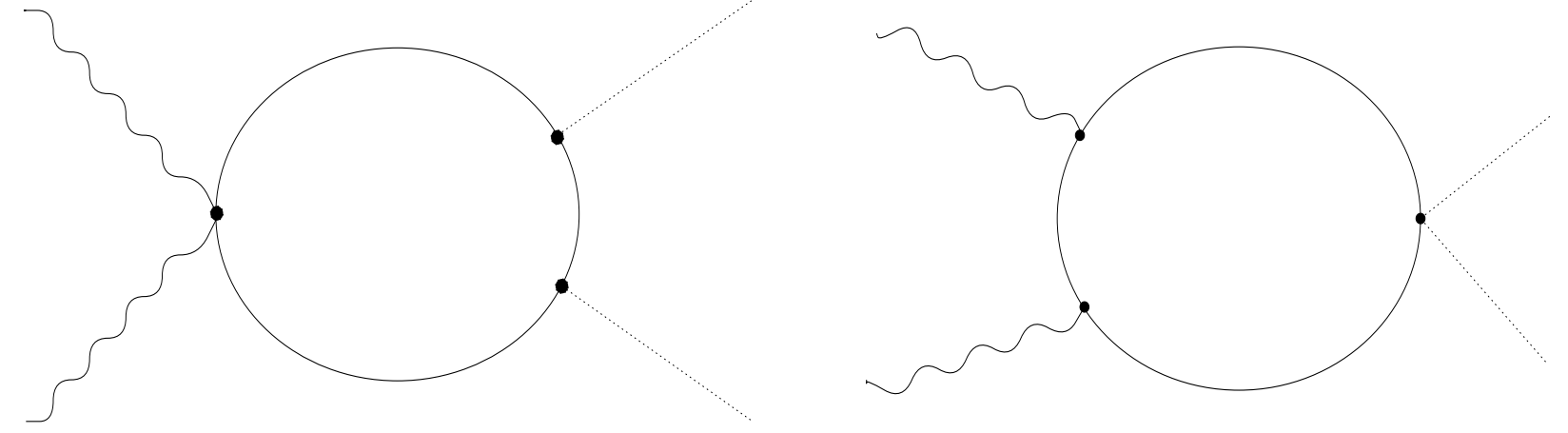

from the action, $\mathcal{A}_{\mathrm{NJL}}$. Here $\mathcal{Q}$ represents the quark charge matrix. The vector (photon) field $v_{\mu}$ is added according to eqs (2.1) and (2.2) while there are no axial sources. In order to compute the pion matrix element of (3.2) we have to expand $\mathcal{A}_{\mathrm{NJL}}$ up to quadratic order in both the pion and photon fields. We note that diagrams like those in figure 3.1, which have identical particles at a single vertex, have no absorptive part in the Bjorken limit since these diagrams depend on only one of the two external momenta. It is therefore sufficient to consider

$$
-\mathbf{D D}_{5}=\partial^{2}+m^{2}+g \gamma_{5}[\not \partial, \vec{\pi} \cdot \tau]-i(\not \partial \mathcal{Q}+\psi \mathcal{Q} \not \partial)+i g \gamma_{5}[\vec{\pi} \cdot \tau, \psi \mathcal{Q}]+\ldots
$$

This expression shows that in the present formulation we are dealing with scalar quarks whose derivative interactions exhibit the spinorial nature of the quarks. Consequently, the substitution of (3.3) into $\mathcal{A}_{\mathrm{R}}$ generates many diagrams. In particular we recognize from eq (3.3) that the present formulation of the NJL model contains vertices which are not expected within a simple Dirac theory and we will have to go beyond computing standard 'handbag' diagrams. First, there are pion-photon contact interactions contributing to diagrams like the one in the left panel of figure 3.2. In addition there are derivative couplings of the pions to the quarks. They are dimension five operators which cause diagrams like that in the right panel of figure 3.2 not to vanish in the Bjorken limit. The diagrams in figure 3.2 have in common the $\left(\vec{\pi} v_{\mu} \vec{\pi} v_{\nu}\right)$ ordering of the pion and photon insertions. The isospin part of the functional trace provides the factor $\operatorname{tr}\left(\tau_{a} \mathcal{Q} \tau_{a} \mathcal{Q}\right)$ where $a$ is the isospin quantum number of the pion. Apparently this leads to isospin violation as the contribution to the structure functions of charged and uncharged pions will be different. Fortunately it turns out that the sum of all diagrams with this ordering vanishes in the Bjorken limit. In addition there are diagrams with the vertex ordering $\left(v_{\mu} \vec{\pi} \vec{\pi} v_{\nu}\right)$. The respective isospin factors $\operatorname{tr}\left(\tau_{a} \tau_{a} \mathcal{Q Q}\right)=\frac{10}{9}$ do not depend on the isospin of the pion. Typical examples are depicted in figure 3.3. Collecting the contributions of all relevant graphs we find

$$
\begin{aligned}
\left\langle\pi(p)\left|\frac{\delta^{2}}{\delta v^{\mu}(\xi) \delta v^{\nu}(0)} \mathcal{A}_{\mathrm{NJL}}[\psi \mathcal{Q}]\right|_{v_{\mu}=0} \mid \pi(p)\right\rangle= \\
\quad \frac{5 g^{2} N_{C}}{18} \sum_{i=0}^{2} c_{i} \int \frac{d^{4} k}{(2 \pi)^{4}} \frac{1}{-k^{2}+m^{2}+\Lambda_{i}^{2}-i \epsilon} \frac{1}{\left[-(k-p)^{2}+m^{2}+\Lambda_{i}^{2}-i \epsilon\right]^{2}}
\end{aligned}
$$


Figure 3.2: Cutting the quark loop according to Cutkosky's rules. External pion and photon lines are indicated by dotted and curly lines. These diagrams have an isospin violating ordering $\left(v_{\mu} \vec{\pi} v_{\nu} \vec{\pi}\right)$.
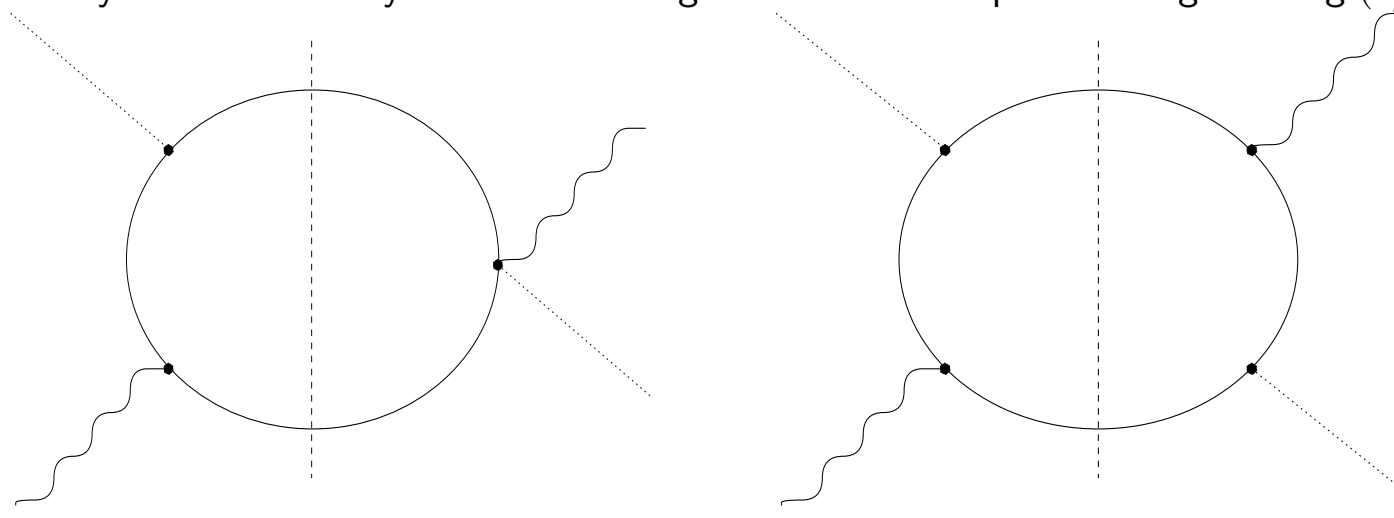

Figure 3.3: Cutting the quark loop according to Cutkosky's rules. External pion and photon lines are indicated by dotted and curly lines, respectively. These diagrams have the proper isospin ordering $\left(v_{\mu} v_{\nu} \vec{\pi} \vec{\pi}\right)$.
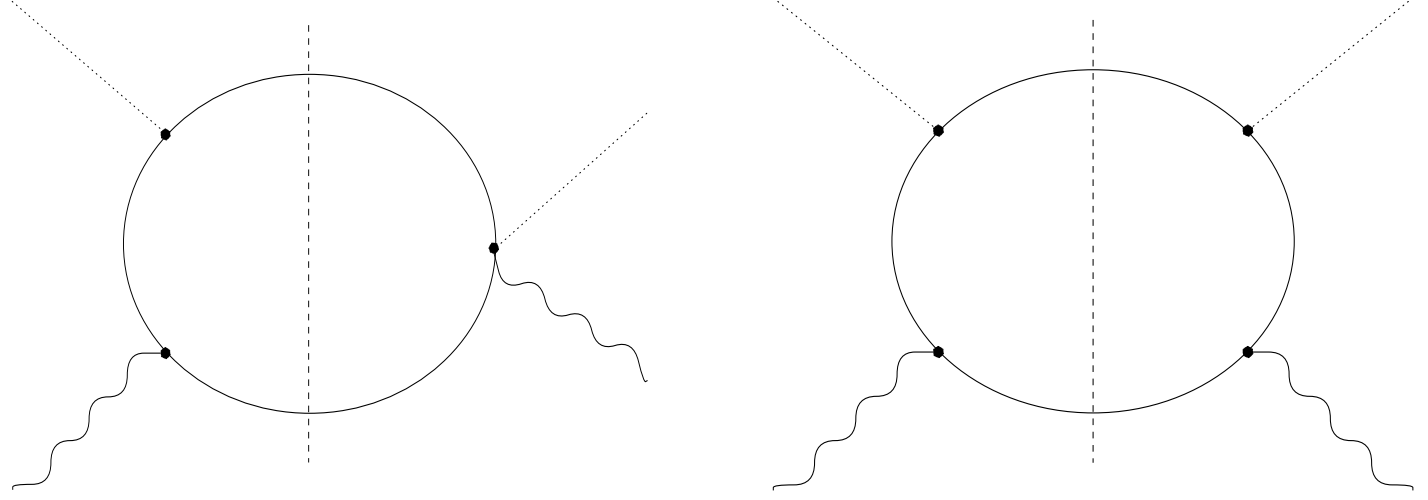

$$
\begin{gathered}
\times\left\{\frac{-(k-p)^{2}+m^{2}+\Lambda_{i}^{2}}{-(k-q-p)^{2}+m^{2}+\Lambda_{i}^{2}-i \epsilon} \operatorname{tr}\left(\not p \gamma^{\mu} \not q \gamma^{\nu}+\not p \gamma^{\nu} \not \gamma^{\mu}\right)\right. \\
+\frac{-(k-p)^{2}+m^{2}+\Lambda_{i}^{2}}{-(k+q-p)^{2}+m^{2}+\Lambda_{i}^{2}-i \epsilon} \operatorname{tr}\left(\not p \gamma^{\mu} \phi \gamma^{\nu}+\not p \gamma^{\nu} \not \phi \gamma^{\mu}\right) \\
+2 m^{2}\left[\frac{\operatorname{tr}\left([\not k-\not p] \gamma^{\mu} \not \gamma^{\nu}\right)}{-(k+q-p)^{2}+m^{2}+\Lambda_{i}^{2}-i \epsilon}\right. \\
\left.\left.-\frac{\operatorname{tr}\left([\not k-\not p] \gamma^{\nu} \not \gamma^{\mu}\right)}{-(k-q-p)^{2}+m^{2}+\Lambda_{i}^{2}-i \epsilon}\right]\right\} .
\end{gathered}
$$

This expression simplifies considerably when applying Cutkosky's rules in the Bjorken limit,

$$
\begin{aligned}
& \frac{1}{-k^{2}+m^{2}+\Lambda_{i}^{2}-i \epsilon} \quad \longrightarrow \quad-2 i \pi \delta\left(k^{2}-m^{2}-\Lambda_{i}^{2}\right), \\
& \frac{1}{-(k \pm q-p)^{2}+m^{2}+\Lambda_{i}^{2}-i \epsilon} \quad \longrightarrow \quad-\frac{i \pi}{q^{-}} \delta\left(q^{+} \pm(k-p)^{+}\right) \text {. }
\end{aligned}
$$

Here we have already employed light-cone coordinates because they render the implementation of the Bjorken limit quite transparent; $q^{-} \rightarrow \infty$ and $q^{+} \rightarrow x p^{+}$. At this point we pause to have 
a second look at the replacement (3.6). In the $\delta$-function all terms which did not multiply $q^{-}$ have dropped out in the Bjorken limit. In particular, all the dependence on the cut-offs, $\Lambda_{i}$, has disappeared. This yields the desired scaling behavior which would not have been recovered in the frequently adopted proper-time regularization [17]. For scaling to occur, a cut-off which is additive to the quark mass appears mandatory.

The pion structure function is now straightforwardly obtained from $T_{11}+T_{22}$,

$$
\begin{gathered}
F(x)=\frac{5}{9}\left(4 N_{C} g^{2}\right) \sum_{i=0}^{2} c_{i} \int \frac{d^{4} k}{(2 \pi)^{4}} \frac{2 \pi \delta\left(k^{2}-m^{2}-\Lambda_{i}^{2}\right)}{\left[-(k-p)^{2}+m^{2}+\Lambda_{i}^{2}-i \epsilon\right]^{2}} \\
\times\left\{\left[-(k-p)^{2}+m^{2}+\Lambda_{i}^{2}\right] p^{+}\left[\delta\left(k^{+}-p^{+}-q^{+}\right)-\delta\left(k^{+}-p^{+}+q^{+}\right)\right]\right. \\
\left.\quad+m^{2}\left(k^{+}-p^{+}\right)\left[\delta\left(k^{+}-p^{+}-q^{+}\right)-\delta\left(k^{+}-p^{+}+q^{+}\right)\right]\right\} .
\end{gathered}
$$

The remaining integrals can be performed yielding

$$
\begin{aligned}
F(x) & =\frac{10}{9} N_{C} g^{2} \sum_{i=0}^{2} c_{i} \int \frac{d^{2} k_{\perp}}{(2 \pi)^{3}} \frac{1}{x(1-x)} \frac{M_{i}^{2}}{\left[M_{i}^{2}-m^{2}\right]^{2}}, \\
M_{i}^{2} & =\frac{1}{x(1-x)}\left[k_{\perp}^{2}+m^{2}+\Lambda_{i}^{2}\right] .
\end{aligned}
$$

Comparing this expression with the definition of the pion polarization function (2.10) it can easily be verified that

$$
F(x)=\left.\frac{5}{9}\left(4 N_{C} g^{2}\right) \frac{d}{d p^{2}}\left[p^{2} \Pi\left(p^{2}, x\right)\right]\right|_{p^{2}=m_{\pi}^{2}} .
$$

This well-known result [17, 31] particularly implies that in the chiral limit $\left(m_{\pi}=0\right)$ the pion structure function equals unity, up to charge normalization. In appendix A we show how this result can also be obtained from the Bethe-Salpeter amplitude (2.10).

\section{Bjorken limit and scaling}

At this point we recognize that the same result could have been obtained by ignoring the issue of regularization and simply computing the 'handbag' diagram (right panel of figure 3.3) using the vertices contained in the simple Dirac operator,

$$
i \not \partial+m+i g \gamma_{5} \vec{\pi} \cdot \vec{\tau}+\psi \mathcal{Q}
$$

and subsequently enforcing the Pauli-Villars regularization on the polarization function $\Pi\left(q^{2}\right)$. Hence, the question arises whether a simple argument exists for the cancellation of all isospin violating diagrams, which may involve dimension five operators, in the current approach. The appearance of these terms actually is an artifact of the simultaneous expansion in the pion and photon fields (3.3). According to eqs (2.1) and (2.2) we might equally well have expanded only in the photon field first (omitting for the time being the trivial factors of the charge matrix)

$$
-\operatorname{Tr}\left\{\left(-\mathbf{D}^{(\pi)} \mathbf{D}_{5}^{(\pi)}+\Lambda_{i}^{2}\right)^{-1}\left[\left(\mathbf{D}^{(\pi)} \psi+\psi \mathbf{D}_{5}^{(\pi)}\right)\left(-\mathbf{D}^{(\pi)} \mathbf{D}_{5}^{(\pi)}+\Lambda_{i}^{2}\right)^{-1}\left(\mathbf{D}^{(\pi)} \psi+\psi \mathbf{D}_{5}^{(\pi)}\right)\right]\right\} .
$$


Here square brackets have been introduced to mark those parts through which the large photon momentum runs. Due to the cyclic properties of the trace this is merely a choice. In momentum space the propagator inside the square brackets behaves like $1 / Q^{2}$ in the Bjorken limit. In particular this implies that

$$
\begin{aligned}
{[\ldots] } & \longrightarrow\left(\mathbf{D}^{(\pi)} \psi+\psi \mathbf{D}_{5}^{(\pi)}\right)\left(-\mathbf{D}^{(\pi)} \mathbf{D}_{5}^{(\pi)}\right)^{-1}\left(\mathbf{D}^{(\pi)} \psi+\psi \mathbf{D}_{5}^{(\pi)}\right) \\
& \longrightarrow-\mathbf{D}^{(\pi)} \psi\left(\mathbf{D}_{5}^{(\pi)}\right)^{-1} \psi-\psi\left(\mathbf{D}^{(\pi)}\right)^{-1} \psi \mathbf{D}_{5}^{(\pi)}
\end{aligned}
$$

This replacement tells us that the propagator through which the large photon momentum runs will not be effected by the regularization. Hence in the Bjorken limit there will be no contributions which behave like $Q^{2} / \Lambda_{i}^{2}$; thereby the proper scaling behavior is manifest. In other regularization schemes, like e.g. proper-time, wherein the cut-off is not additive to the loop momenta, the absence of such scaling violating contributions is not apparent. In the above expression we have also omitted terms which contained a two photon vertex or were suppressed by additional factors of $1 / Q^{2}$. Previously we expanded the operator (4.1) in powers of the pion field leading to complicated three and four vertex quark loops as in figure 3.2. Now we see that the Bjorken limit enforces the above observed cancellations among those diagrams. The expression (4.2) simplifies even further by noting that the quark propagator inside the photon insertions carries the large photon momentum and should hence be approximated by the free massless propagator,

$$
[\cdots] \longrightarrow \mathbf{D}^{(\pi)} \psi(\not \partial)^{-1} \psi-\psi(\not \partial)^{-1} \psi \mathbf{D}_{5}^{(\pi)}
$$

Together with the propagator in (4.1) this will yield the standard 'handbag' diagram with the propagators connecting the quark-pion and quark-photon vertices regularized according to the Pauli-Villars scheme. The transition from the expression (4.1) to (4.3) is illustrated in figure 4.1. One may still wonder whether the separation into (infinitely) large and small momentum components of the expression (4.1) would be arbitrary, after all the functional trace implies the integration over all momenta. For this purpose assume that the momenta flowing through the two propagators in the functional trace (4.1) would both be comparable to the photon momentum $Q^{2}$. Then also the propagator outside the square brackets can be approximated by the free massless propagator and the object (4.1) simplifies even further,

$$
2 \operatorname{Tr}\left\{(\not \partial)^{-1} \psi(\not \partial)^{-1} \psi\right\} .
$$

This will contribute a disconnected graph whose $q$-dependence is completely given by

$$
\left(2 q^{\mu} q^{\nu}-q^{2} g^{\mu \nu}\right) \frac{1}{q^{2}+i \epsilon} \frac{1}{q^{2}+i \epsilon} .
$$

This contribution vanishes like $1 / q^{2}$ in the Bjorken limit. The fact that the external momenta (pion or nucleon versus photon) are on completely different scales allows us to distinguish the quark propagators accordingly. We could furthermore ask for possible corrections to the expression (4.3) when taking the quark propagator carrying $q$ not to be the free massless one. 
Figure 4.1: Cutting the quark loop. The thick lines denote quark propagators which contain the full dependence on the pion fields. As in figure 3.2 thin lines refer to free quark propagators carrying the infinite photon momentum $q$. The arrow indicates the Bjorken limit.

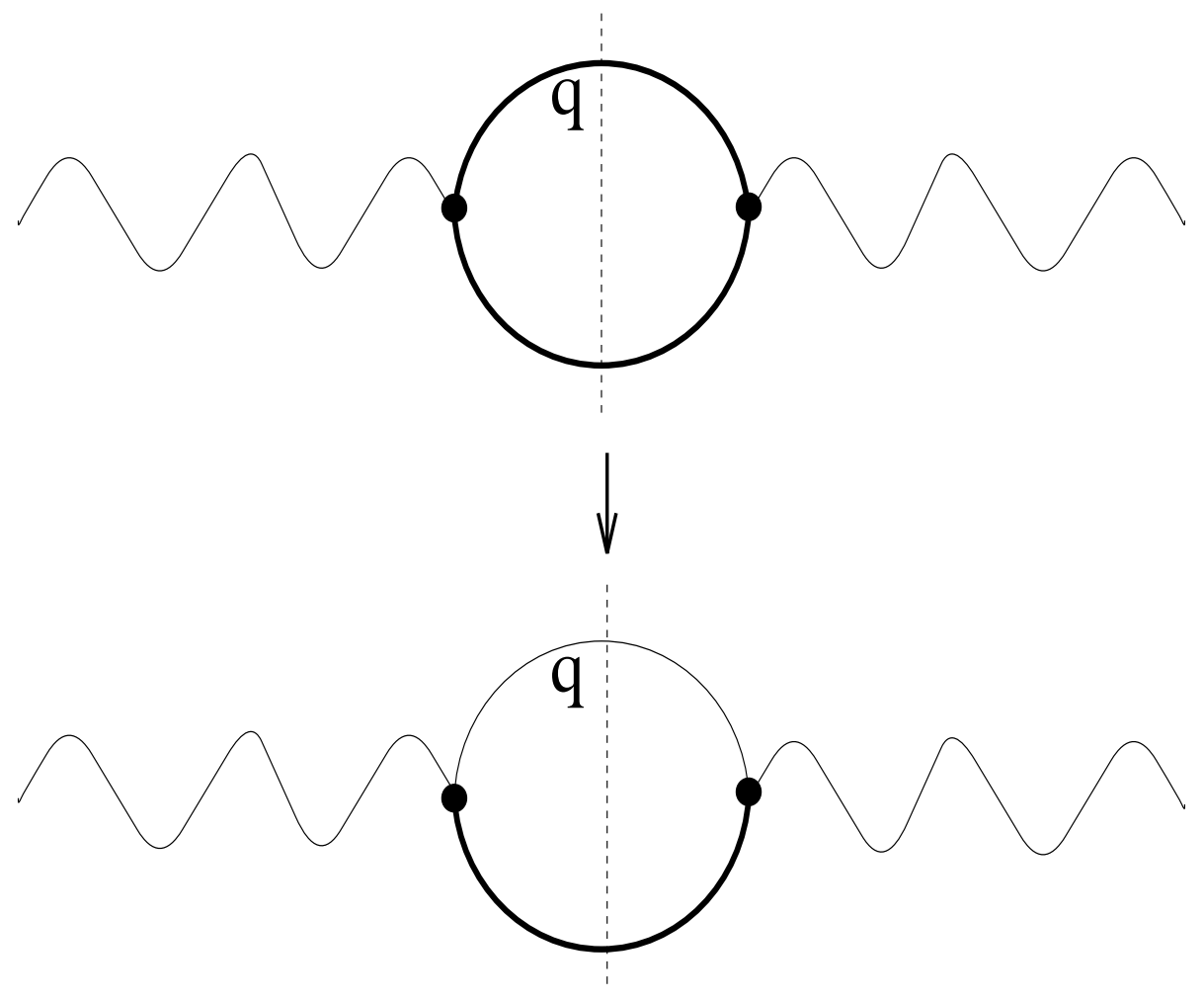

These corrections would come about as an expansion in the pion field contained in the Dirac operators $\mathbf{D}^{(\pi)}$ and $\mathbf{D}_{5}^{(\pi)}$. The first order correction is shown in figure 4.2. As a matter of fact this graph is similar to the one in the right panel of figure 3.2 with non-trivial quark propagators on either side of the photon vertices. Once we have organized the expansion such that the dimension-five operators no longer contribute (at the end they should cancel anyhow), such diagrams will be suppressed in the Bjorken limit. Thus the replacement in (4.3) is indeed exact in the Bjorken limit. In appendix B we discuss this replacement in the context of the Wigner transformation.

Having clarified these issues for the pion structure functions we now turn to the case of the nucleon. After re-introducing the charge matrix factors it will be sufficient to differentiate

$$
\mathcal{A}_{\Lambda, \mathrm{R}}^{(2, v)}=-i \frac{N_{C}}{4} \sum_{i=0}^{2} c_{i} \operatorname{Tr}\left\{\left(-\mathbf{D}^{(\pi)} \mathbf{D}_{5}^{(\pi)}+\Lambda_{i}^{2}\right)^{-1}\left[\mathcal{Q}^{2} \psi(\not \partial)^{-1} \psi \mathbf{D}_{5}^{(\pi)}-\mathbf{D}^{(\pi)}\left(\psi(\not \partial)^{-1} \psi\right)_{5} \mathcal{Q}^{2}\right]\right\}
$$

with respect to the vector sources in order to obtain the Compton amplitude in the Bjorken limit.

At this point it is important to explain the crucial role of the subscript ' 5 ' attached to the second term in square brackets of eq (4.6). For this second term we have to recall that 
Figure 4.2: The first order perturbation to the lower part of figure 4.1 in expanding with respect to the pion field which is denoted by the dotted line.

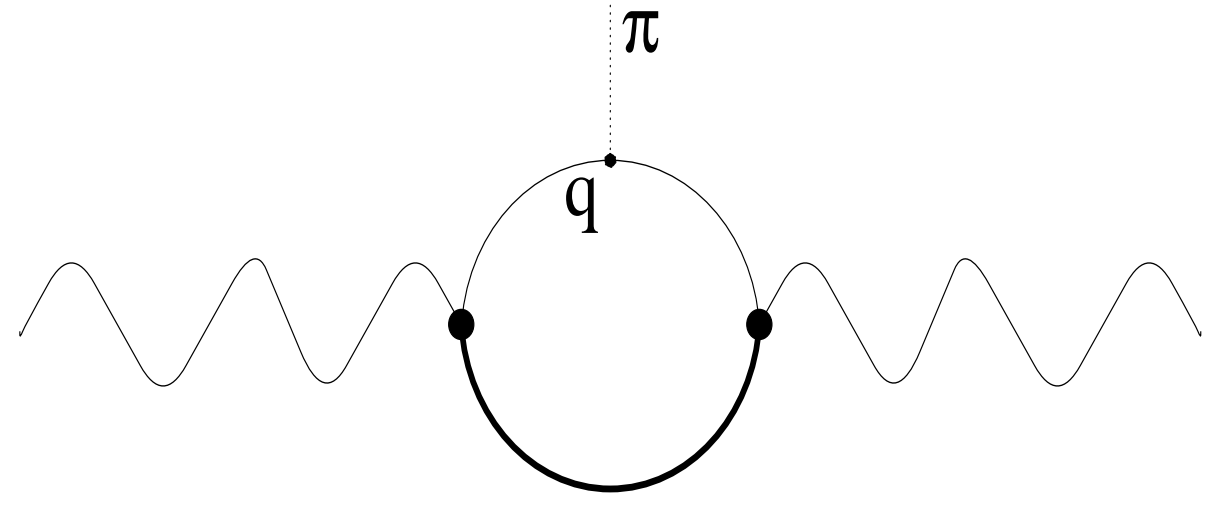

the (inverse) derivative operator in $\psi(\not \partial)^{-1} \psi$ is actually associated with the expansion of $\mathbf{D}_{5}$. When comparing this $\gamma_{5}$ odd operator to the ordinary Dirac operator in eqs (2.1) and (2.2) one observes immediately that $\mathbf{D}_{5}$ has a relative sign between the derivative operator $i \not \partial$ and the axial source $\not \gamma \gamma_{5}$. In section 6 we will show that for the regularization of the structure functions to be consistent with the sum rules this relative sign must also be reflected in the Dirac decomposition of $\left(\psi(\not \partial)^{-1} \psi\right)_{5}$. Therefore the axial-vector component of $\left(\psi(\not \partial)^{-1} \psi\right)_{5}$ requires a relative sign. With $S_{\mu \rho \nu \sigma}=g_{\mu \rho} g_{\nu \sigma}+g_{\rho \nu} g_{\mu \sigma}-g_{\mu \nu} g_{\rho \sigma}$, that is

$$
\gamma_{\mu} \gamma_{\rho} \gamma_{\nu}=S_{\mu \rho \nu \sigma} \gamma^{\sigma}-i \epsilon_{\mu \rho \nu \sigma} \gamma^{\sigma} \gamma^{5} \quad \text { while } \quad\left(\gamma_{\mu} \gamma_{\rho} \gamma_{\nu}\right)_{5}=S_{\mu \rho \nu \sigma} \gamma^{\sigma}+i \epsilon_{\mu \rho \nu \sigma} \gamma^{\sigma} \gamma^{5}
$$

The fact that the sum rules enforce this extension of the regularization scheme is not all surprising when noting that the derivative operator $i \not \partial$ fixes the Noether currents. Rather it is imposed and a consequence of the 'sum rules' of the model defined by $\mathbf{D}_{5}, c f$. appendix $\mathrm{C}$. We recall that the $\mathbf{D}_{5}$ model, which is not physical, has been introduced as a device to allow for a regularization which maintains the anomaly structure of the underlying theory. Hence it is not at all surprising that further specification of this regularization prescription is demanded in order to formulate a fully consistent model. It should be stressed that this issue is not specific to the Pauli-Villars scheme but rather all schemes which do regularize the sum, $\log (\mathbf{D})+\log \left(\mathbf{D}_{5}\right)$ but not the difference, $\log (\mathbf{D})-\log \left(\mathbf{D}_{5}\right)$ will require the specification (4.7). Since only the polarized, i.e. spin dependent, structure functions are effected, this issue has not shown up when computing the pion structure functions in the Pauli-Villars scheme.

For the imaginary part of the action the expression analogous to (4.6) reads

$$
\mathcal{A}_{\Lambda, \mathrm{I}}^{(2, v)}=-i \frac{N_{C}}{4} \operatorname{Tr}\left\{\left(-\mathbf{D}^{(\pi)} \mathbf{D}_{5}^{(\pi)}\right)^{-1}\left[\mathcal{Q}^{2} \psi(\not \partial)^{-1} \psi \mathbf{D}_{5}^{(\pi)}+\mathbf{D}^{(\pi)}\left(\psi(\not \partial)^{-1} \psi\right)_{5} \mathcal{Q}^{2}\right]\right\} .
$$

Again, it is understood that the large photon momentum runs only through the operators in square brackets.

It is also noticed that in the unregularized case $\left(\Lambda_{i} \equiv 0\right)$ the contributions associated to the expansion of $\mathbf{D}_{5}$ cancel in the sum (4.6) and (4.8) leaving

$$
\mathcal{A}^{(2, v)}=i \frac{N_{C}}{2} \operatorname{Tr}\left\{\left(\mathbf{D}^{(\pi)}\right)^{-1}\left[\mathcal{Q}^{2} \psi(\not \partial)^{-1} \psi\right]\right\} \text {. }
$$


This expression generates the 'handbag' diagram upon expansion in the pion field. A similar cancellation would occur if the imaginary part were also regularized. In particular, the $\gamma_{5}$-odd pieces stemming from expanding $\mathbf{D}_{5}$ would cancel. With these remarks on the regularization of the Compton tensor in the Bjorken limit we are prepared to proceed with the full soliton calculation.

\section{The nucleon structure functions}

Here we will only discuss the contribution of the polarized vacuum to the nucleon structure functions. The contribution of the distinct valence level, which is not effected by the regularization, $c f$. eq (2.17), has previously been detailed [3, 10].

Before carrying out a calculation like that in section 3 we must define the hadronic tensor for localized field configurations. Among other things this demands the restoration of translational invariance. This is accomplished by introducing a collective coordinate, $\vec{R}$, which describes the position of a soliton (nucleon) [32] with its momentum, $\vec{p}$ being conjugate to this collective coordinate, i.e. $\langle\vec{R} \mid \vec{p}\rangle=\sqrt{2 E} \exp (i \vec{R} \cdot \vec{p})$. Here $E=\sqrt{\vec{p}^{2}+M_{N}^{2}}$ denotes the nucleon energy. The Compton amplitude (1.8) is then obtained by taking the pertinent matrix element and averaging over the position of the soliton,

$$
\begin{aligned}
T_{\mu \nu}^{a b} & =2 i M_{N} \int d^{4} \xi \int d^{3} R e^{i q \cdot \xi}\left\langle p, s\left|T\left\{J_{\mu}^{a}(\xi-R) J_{\nu}^{b \dagger}(-R)\right\}\right| p, s\right\rangle \\
& =2 i M_{N} \int d^{4} \xi_{1} \int d^{3} \xi_{2} e^{i q \cdot\left(\xi_{1}-\xi_{2}\right)}\left\langle s\left|T\left\{J_{\mu}^{a}\left(\xi_{1}\right) J_{\nu}^{b \dagger}\left(\xi_{2}\right)\right\}\right| s\right\rangle
\end{aligned}
$$

Here we have made use of the fact that the initial and final nucleon states have identical momenta. Furthermore we will treat $\xi_{2}$ as a four-vector keeping in mind that its temporal component vanishes, $\xi_{2}^{0}=0$. Finally the spin-flavor matrix elements will be evaluated in the space of the collective coordinates $A$, which have been introduced in eq (2.18).

\section{5a. The unregularized case}

Before going into details we wish to briefly discuss the formal result associated with the unregularized case (4.9) as a warm-up. According to eq (5.1) we evaluate

$$
\begin{aligned}
T\left(J_{\mu}\left(\xi_{1}\right), J_{\nu}\left(\xi_{2}\right)\right)= & \left.i N_{C} \frac{\delta^{2}}{\delta v^{\mu}\left(\xi_{1}\right) \delta v^{\nu}\left(\xi_{2}\right)} \operatorname{Tr}\left\{\left(-\mathbf{D}^{(\pi)}\right)^{-1}\left[\mathcal{Q}^{2} \psi(\not \partial)^{-1} \not\right]\right\}\right|_{v_{\mu}=0} \\
= & i \frac{N_{C}}{2} \operatorname{Tr}\left\{( - \mathbf { D } ^ { ( \pi ) } ) ^ { - 1 } \mathcal { Q } ^ { 2 } \left[\gamma_{\mu} \delta^{4}\left(\hat{x}-\xi_{1}\right)(\not \partial)^{-1} \gamma_{\nu} \delta^{4}\left(\hat{x}-\xi_{2}\right)\right.\right. \\
& \left.\left.+\gamma_{\nu} \delta^{4}\left(\hat{x}-\xi_{2}\right)(\not \partial)^{-1} \gamma_{\mu} \delta^{4}\left(\hat{x}-\xi_{1}\right)\right]\right\} .
\end{aligned}
$$

Here $\hat{x}$ refers to the position operator. Its introduction represents a suitable tool to evaluate the functional trace because $\hat{x}|x\rangle=x|x\rangle$. This functional trace is computed by using a plane-wave basis for the operator in the square brackets while the matrix elements of $\mathbf{D}^{(\pi)}$ are evaluated employing the eigenfunctions $\Psi_{\alpha}$ of the Dirac Hamiltonian (2.16). Confining ourselves to the 
leading order in $1 / N_{C}$ piece, i.e. omitting, for the time being, the corrections due to the collective rotations yields

$$
\begin{aligned}
T_{\mu \nu}(q)=-M_{N} N_{C} \int \frac{d \omega}{2 \pi} \sum_{\alpha} \int d^{4} \xi_{1} \int d^{3} \xi_{2} \int \frac{d^{4} k}{(2 \pi)^{4}} \mathrm{e}^{i \xi_{1}^{0}\left(q^{0}+k^{0}\right)} \mathrm{e}^{-i\left(\vec{\xi}_{1}-\vec{\xi}_{2}\right) \cdot(\vec{q}+\vec{k})} \frac{1}{k^{2}+i \epsilon} \\
\times \frac{\omega+\epsilon_{\alpha}}{\omega^{2}-\epsilon_{\alpha}^{2}+i \epsilon}\langle N|\left\{\bar{\Psi}_{\alpha}\left(\vec{\xi}_{1}\right) \mathcal{Q}_{A}^{2} \gamma_{\mu} \not k \gamma_{\nu} \Psi_{\alpha}\left(\vec{\xi}_{2}\right) \mathrm{e}^{i \xi_{1}^{0} \omega}\right. \\
\left.-\bar{\Psi}_{\alpha}\left(\vec{\xi}_{2}\right) \mathcal{Q}_{A}^{2} \gamma_{\nu} \not k \gamma_{\nu} \Psi_{\alpha}\left(\vec{\xi}_{1}\right) \mathrm{e}^{-i \xi_{1}^{0} \omega}\right\}|N\rangle .
\end{aligned}
$$

A few remarks are in order. First, the frequency integral stems from summing over the eigenvalues of $i \partial_{t}$ contained in $\mathbf{D}^{(\pi)}$ in the limit of an infinitely large time interval (in Euclidean space or with Feynman boundary conditions). This large time interval singles out the vacuum contribution to the functional trace 28]. Second, the momentum integral over $k$ essentially introduces the Fourier transformation of the single quark wave-functions $\Psi_{\alpha}$ which originated from the projection of these states on the elements of the plane wave-basis. Note also that the Dirac matrix $\beta$, contained in the definition (2.14) re-assembled to the adjoint spinor $\bar{\Psi}_{\alpha}=\Psi_{\alpha}^{\dagger} \beta$. As we will see, this need not be the case in the fully regularized treatment. The collective coordinates, $A$ are only treated at the leading order in $1 / N_{C}$ by rotating the charge matrix $\mathcal{Q}^{2} \rightarrow \mathcal{Q}_{A}^{2}=A^{\dagger} \mathcal{Q}^{2} A$ and taking the nucleon matrix elements in the space of the collective coordinates (2.21). The next-to-leading order can straightforwardly be accounted for by expanding the matrix elements of the operator (2.19),

$$
\left\langle\omega, \alpha\left|\left(\mathbf{D}^{(\pi)}\right)^{-1}\right| \omega, \beta\right\rangle=\frac{\delta_{\alpha \beta}}{\omega-\epsilon_{\alpha}}+\frac{1}{\omega-\epsilon_{\alpha}}\langle\alpha|\vec{\tau} \cdot \vec{\Omega}| \beta\rangle \frac{1}{\omega-\epsilon_{\beta}}+\mathcal{O}\left(\vec{\Omega}^{2}\right) .
$$

In addition there will be $1 / N_{C}$ corrections associated with the bi-locality in the time coordinates $\xi_{1}^{0}=t$ and $\xi_{2}^{0}=0$. This bi-locality not only effects the eigen-functions of $i \partial_{t}$ but also the collective coordinates. However, it can be rewritten as a local quantity by expanding [13, 14, 33]

$$
\begin{aligned}
\langle t, \vec{\xi}|A(\hat{t})| \omega, \alpha\rangle & =A(t) \mathrm{e}^{-i \omega t} \Psi_{\alpha}(\vec{\xi}) \\
& =A(0)\left[1+\frac{i t}{2} \vec{\tau} \cdot \vec{\Omega}\right] \mathrm{e}^{-i \omega t} \Psi_{\alpha}(\vec{\xi})+\mathcal{O}\left(\vec{\Omega}^{2}\right)
\end{aligned}
$$

Apparently the non-linearity in the time-coordinate becomes feasible in the large $N_{C}$ expansion since the angular velocity, $\vec{\Omega}$ is of order $1 / N_{C}$, cf. eq (2.20). We will see later that the contribution to the structure functions stemming from the $t$-dependent coefficient of the angular velocity in (5.5) is most conveniently expressed as a derivative with respect to Bjorken $x$. As the induced terms (5.4) and (5.5) do not depend on the photon momentum, $q$, their inclusion in the Compton amplitude is just a matter of book-keeping rather than a principle obstacle.

Putting the quark fields on-shell according to Cutkosky's rules identifies the imaginary part 
of the Compton amplitude and hence the hadronic tensor

$$
\begin{aligned}
W_{\mu \nu}=M_{N} & N_{C} \sum_{\alpha} \operatorname{sign}\left(\epsilon_{\alpha}\right) \int d t \int d^{3} \xi_{1} \int d^{3} \xi_{2} \int \frac{d^{4} k}{(2 \pi)^{4}} \mathrm{e}^{i t\left(q^{0}+k^{0}\right)} \mathrm{e}^{-i\left(\vec{\xi}_{1}-\vec{\xi}_{2}\right) \cdot(\vec{q}+\vec{k})} \delta\left(k^{2}\right) k^{\rho} \\
\times & \langle N| S_{\mu \rho \nu \sigma}\left\{\bar{\Psi}_{\alpha}\left(\vec{\xi}_{1}\right) \mathcal{Q}_{A}^{2} \gamma^{\sigma} \Psi_{\alpha}\left(\vec{\xi}_{2}\right) \mathrm{e}^{i \epsilon_{\alpha} t}-\bar{\Psi}_{\alpha}\left(\vec{\xi}_{2}\right) \mathcal{Q}_{A}^{2} \gamma^{\sigma} \Psi_{\alpha}\left(\vec{\xi}_{1}\right) \mathrm{e}^{-i \epsilon_{\alpha} t}\right\} \\
& -i \epsilon_{\mu \rho \nu \sigma}\left\{\bar{\Psi}_{\alpha}\left(\vec{\xi}_{1}\right) \mathcal{Q}_{A}^{2} \gamma^{\sigma} \gamma_{5} \Psi_{\alpha}\left(\vec{\xi}_{2}\right) \mathrm{e}^{i \epsilon_{\alpha} t}+\bar{\Psi}_{\alpha}\left(\vec{\xi}_{2}\right) \mathcal{Q}_{A}^{2} \gamma^{\sigma} \gamma_{5} \Psi_{\alpha}\left(\vec{\xi}_{1}\right) \mathrm{e}^{-i \epsilon_{\alpha} t}\right\}|N\rangle .
\end{aligned}
$$

It is recognized that this expression for the hadronic tensor is analogous to the one obtained in the bag model some time ago [3]. For the purpose of comparison we have introduced the time coordinate $t=\xi_{1}^{0}$. Although the nature of the quark spinors in the NJL and bag models is quite different, this formal equality is not surprising because all that has entered so far, have been the properties of the symmetry currents in the model. Moreover, in both models these currents are formally identical to currents of a non-interacting Dirac theory. At this order in $1 / N_{C}$ the only difference is contained in the labels " $\alpha$ " which characterize the single quark levels.

We also recognize two different contributions to the hadronic tensor: one associated with forward moving intermediate quarks $\left(\xi_{1}, \mu \rightarrow \xi_{2}, \nu\right)$ and one wherein those quarks propagate backward $\left(\xi_{2}, \nu \rightarrow \xi_{1}, \mu\right)$. In the parton model language these two pieces correspond to quark and anti-quark distributions 円, respectively.

\section{5b. The fully regularized case}

Here we compute the contribution to the hadronic tensor stemming from the time-ordered product in (4.6). It is just a matter of generalizing the previous calculation to obtain the contribution of the regularized real part, $\mathcal{A}_{\mathrm{R}}$ to the hadronic tensor. For the time being we will confine ourselves to the leading order contribution in $1 / N_{C}$ while the resulting formulas including the cranking corrections are presented in appendix D. We find

$$
\begin{aligned}
& T_{\mu \nu}^{(\mathrm{R})}=-M_{N} \frac{N_{C}}{2} \int \frac{d \omega}{2 \pi} \sum_{\alpha} \int d t \int d^{3} \xi_{1} \int d^{3} \xi_{2} \int \frac{d^{4} k}{(2 \pi)^{4}} \mathrm{e}^{i\left(q^{0}+k^{0}\right) t} \mathrm{e}^{-i\left(\vec{\xi}_{1}-\vec{\xi}_{2}\right) \cdot(\vec{q}+\vec{k})} \frac{1}{k^{2}+i \epsilon} \\
& \times\langle N| \sum_{i=0}^{2} c_{i}\left\{\frac{\omega+\epsilon_{\alpha}}{\omega^{2}-\epsilon_{\alpha}^{2}-\Lambda_{i}^{2}+i \epsilon}\left[\Psi_{\alpha}^{\dagger}\left(\vec{\xi}_{1}\right) \beta \mathcal{Q}_{A}^{2} \gamma_{\mu} \not k \gamma_{\nu} \Psi_{\alpha}\left(\vec{\xi}_{2}\right) \mathrm{e}^{i \omega t}-\Psi_{\alpha}^{\dagger}\left(\vec{\xi}_{2}\right) \beta \mathcal{Q}_{A}^{2} \gamma_{\nu} \not k \gamma_{\mu} \Psi_{\alpha}\left(\vec{\xi}_{1}\right) \mathrm{e}^{-i \omega t}\right]\right. \\
& \left.+\frac{\omega-\epsilon_{\alpha}}{\omega^{2}-\epsilon_{\alpha}^{2}-\Lambda_{i}^{2}+i \epsilon}\left[\Psi_{\alpha}^{\dagger}\left(\vec{\xi}_{1}\right) \mathcal{Q}_{A}^{2}\left(\gamma_{\mu} \not k \gamma_{\nu}\right)_{5} \beta \Psi_{\alpha}\left(\vec{\xi}_{2}\right) \mathrm{e}^{i \omega t}-\Psi_{\alpha}^{\dagger}\left(\vec{\xi}_{2}\right) \mathcal{Q}_{A}^{2}\left(\gamma_{\nu} \not k \gamma_{\mu}\right)_{5} \beta \Psi_{\alpha}\left(\vec{\xi}_{1}\right) \mathrm{e}^{-i \omega t}\right]\right\}|N\rangle .
\end{aligned}
$$

The first term in curly brackets corresponds the previous result (5.3) in the limit of vanishing regularization. The second term, however, is new and originates from constructing the real part by adding the $\mathbf{D}_{5}$ model. It is also noticed that in this term the Dirac matrix $\beta$ does not combine to the adjoint spinor $\bar{\Psi}_{\alpha}$. Before applying Cutkosky's rules to (5.7) in order to extract the hadronic tensor, we will compute the piece stemming from the time-ordered product in

\footnotetext{
${ }^{1}$ It should be recalled that presently the quark field operators refer to the degrees of freedom in the NJL model. Hence such distributions may not necessarily be identical to those of the QCD current quarks.
} 
(4.8) which is associated to the imaginary part of the bosonized NJL model action. Also in this case, the cranking corrections are relegated to appendix D. In leading order $1 / N_{C}$ the imaginary part of the action contributes

$$
\begin{aligned}
T_{\mu \nu}^{(\mathrm{I})} & =-M_{N} \frac{N_{C}}{2} \int \frac{d \omega}{2 \pi} \sum_{\alpha} \int d t \int d^{3} \xi_{1} \int d^{3} \xi_{2} \int \frac{d^{4} k}{(2 \pi)^{4}} \mathrm{e}^{i\left(q^{0}+k^{0}\right) t} \mathrm{e}^{-i\left(\vec{\xi}_{1}-\vec{\xi}_{2}\right) \cdot(\vec{q}+\vec{k})} \frac{1}{k^{2}+i \epsilon} \\
& \times\langle N|\left\{\frac{\omega+\epsilon_{\alpha}}{\omega^{2}-\epsilon_{\alpha}^{2}+i \epsilon}\left[\Psi_{\alpha}^{\dagger}\left(\vec{\xi}_{1}\right) \beta \mathcal{Q}_{A}^{2} \gamma_{\mu} \not \gamma_{\nu} \Psi_{\alpha}\left(\vec{\xi}_{2}\right) \mathrm{e}^{i \omega t}-\Psi_{\alpha}^{\dagger}\left(\vec{\xi}_{2}\right) \beta \mathcal{Q}_{A}^{2} \gamma_{\nu} \not k \gamma_{\mu} \Psi_{\alpha}\left(\vec{\xi}_{1}\right) \mathrm{e}^{-i \omega t}\right]\right. \\
& \left.-\frac{\omega-\epsilon_{\alpha}}{\omega^{2}-\epsilon_{\alpha}^{2}+i \epsilon}\left[\Psi_{\alpha}^{\dagger}\left(\vec{\xi}_{1}\right) \mathcal{Q}_{A}^{2}\left(\gamma_{\mu} \not k \gamma_{\nu}\right)_{5} \beta \Psi_{\alpha}\left(\vec{\xi}_{2}\right) \mathrm{e}^{i \omega t}-\Psi_{\alpha}^{\dagger}\left(\vec{\xi}_{2}\right) \mathcal{Q}_{A}^{2}\left(\gamma_{\nu} \not k \gamma_{\mu}\right)_{5} \beta \Psi_{\alpha}\left(\vec{\xi}_{1}\right) \mathrm{e}^{-i \omega t}\right]\right\}|N\rangle
\end{aligned}
$$

to the Compton tensor. Except for the regularization this term differs from (5.7) in the sign of the second term. Of course, if the regularization of $T_{\mu \nu}^{(\mathrm{R})}$ were omitted, the sum of (5.7) and (5.8) would combine to (5.3).

Now we can put pieces together providing the full Compton amplitude in the Bjorken limit

$$
\begin{aligned}
T_{\mu \nu}(q) & =-M_{N} \frac{N_{C}}{2} \int \frac{d \omega}{2 \pi} \sum_{\alpha} \int d t \int d^{3} \xi_{1} \int d^{3} \xi_{2} \int \frac{d^{4} k}{(2 \pi)^{4}} \mathrm{e}^{i\left(q_{0}+k_{0}\right) t} \mathrm{e}^{-i(\vec{q}+\vec{k}) \cdot\left(\vec{\xi}_{1}-\vec{\xi}_{2}\right)} \frac{1}{k^{2}+i \epsilon} \\
\times & \langle N|\left\{\left[\mathrm{e}^{i \omega t} \Psi_{\alpha}^{\dagger}\left(\vec{\xi}_{1}\right) \beta \mathcal{Q}_{A}^{2} \gamma_{\mu} \not k \gamma_{\nu} \Psi_{\alpha}\left(\vec{\xi}_{2}\right)-\mathrm{e}^{-i \omega t} \Psi_{\alpha}^{\dagger}\left(\vec{\xi}_{2}\right) \beta \mathcal{Q}_{A}^{2} \gamma_{\nu} \not k \gamma_{\mu} \Psi_{\alpha}\left(\vec{\xi}_{1}\right)\right] f_{\alpha}^{+}(\omega)\right. \\
& \left.+\left[\mathrm{e}^{i \omega t} \Psi_{\alpha}^{\dagger}\left(\vec{\xi}_{1}\right) \mathcal{Q}_{A}^{2}\left(\gamma_{\mu} \not k \gamma_{\nu}\right)_{5} \beta \Psi_{\alpha}\left(\vec{\xi}_{2}\right)-\mathrm{e}^{-i \omega t} \Psi_{\alpha}^{\dagger}\left(\vec{\xi}_{2}\right) \mathcal{Q}_{A}^{2}\left(\gamma_{\nu} \not k \gamma_{\mu}\right)_{5} \beta \Psi_{\alpha}\left(\vec{\xi}_{1}\right)\right] f_{\alpha}^{-}(\omega)\right\}|N\rangle,
\end{aligned}
$$

with the spectral functions

$$
f_{\alpha}^{ \pm}(\omega)=\sum_{i=0}^{2} c_{i} \frac{\omega \pm \epsilon_{\alpha}}{\omega^{2}-\epsilon_{\alpha}^{2}-\Lambda_{i}^{2}+i \epsilon} \pm \frac{\omega \pm \epsilon_{\alpha}}{\omega^{2}-\epsilon_{\alpha}^{2}+i \epsilon}
$$

It is clear that in case we had chosen to regularize $\mathcal{A}_{\mathrm{I}}$ similarly to $\mathcal{A}_{\mathrm{R}}$ the terms involving $f_{\alpha}^{-}(\omega)$ would disappear and consequently the prescription (4.7), associated with the spin dependent part of $\mathbf{D}_{5}$, would not have been required.

As the quark wave-functions are localized, the coordinate space integrals gather most of their support when $\xi_{\sigma}^{1} \sim \xi_{\sigma}^{2}$. Hence only that region of the $k$-integral will be relevant which has $k_{\sigma} \sim-q_{\sigma}$, i.e. the loop momentum should be of the order of the infinitely large photon momentum. Having noted that, we again apply Cutkosky's rules which yields the object of desire, the hadronic tensor. The temporal integral yields $k_{0}=q_{0} \pm \omega$ which then also fixes the spatial part of the loop momentum due to $\delta\left(k^{2}\right)$. To further exploit the Bjorken limit it is useful to introduce the Fourier transformation of the quark wave-functions,

$$
\tilde{\Psi}_{\alpha}(\vec{p})=\int \frac{d^{3} x}{4 \pi} \Psi_{\alpha}(\vec{x}) \mathrm{e}^{i \vec{x} \cdot \vec{p}}
$$

and make use of the fact that the eigenstates of the Dirac Hamiltonian carry definite parity. The latter property allows us to compensate spatial reflections by factors of $\beta$. With these 
preliminaries the spatial integrals can be performed

$$
\begin{aligned}
W_{\mu \nu}(q)= & i M_{N} \frac{N_{C}}{2}(4 \pi)^{2} \int \frac{d \omega}{2 \pi} \sum_{\alpha} \int \frac{d^{3} k}{(2 \pi)^{2}} \frac{1}{2|\vec{k}|} \\
\times\langle N|\left\{\left[\tilde{\Psi}_{\alpha}^{\dagger}(\vec{q}+\vec{k}) \mathcal{Q}_{A}^{2} \beta \gamma_{\mu} \not k \gamma_{\nu} \tilde{\Psi}_{\alpha}(\vec{q}+\vec{k}) \delta\left(|\vec{k}|-q_{0}-\omega\right)\right.\right. & \left.\quad-\tilde{\Psi}_{\alpha}^{\dagger}(\vec{q}+\vec{k}) \mathcal{Q}_{A}^{2} \gamma_{\nu} \not k \gamma_{\mu} \beta \tilde{\Psi}_{\alpha}(\vec{q}+\vec{k}) \delta\left(|\vec{k}|-q_{0}+\omega\right)\right]\left.f_{\alpha}^{+}(\omega)\right|_{\text {pole }} \\
+ & {\left[\tilde{\Psi}_{\alpha}^{\dagger}(\vec{q}+\vec{k}) \mathcal{Q}_{A}^{2}\left(\gamma_{\mu} \not k \gamma_{\nu}\right)_{5} \beta \tilde{\Psi}_{\alpha}(\vec{q}+\vec{k}) \delta\left(|\vec{k}|-q_{0}-\omega\right)\right.} \\
& \left.\quad-\tilde{\Psi}_{\alpha}^{\dagger}(\vec{q}+\vec{k}) \mathcal{Q}_{A}^{2} \beta\left(\gamma_{\nu} \not k \gamma_{\mu}\right)_{5} \tilde{\Psi}_{\alpha}(\vec{q}+\vec{k}) \delta\left(|\vec{k}|-q_{0}+\omega\right)\right]\left.f_{\alpha}^{-}(\omega)\right|_{\text {pole }}|N\rangle .
\end{aligned}
$$

The subscript 'pole' indicates that the spectral integral is restricted to those values of $\omega$ which cause the respective denominators of the spectral functions (5.10) to vanish. It is interesting to remark that those (unphysical) poles which explicitly contain the cut-off, $\Lambda_{i}$, occur in positive and negative pairs. Hence there is no clear distinction between quark and anti-quark contributions. Note that the second term in (5.12) not only differs from the first one in the spectral function but also with regard to the position of the Dirac matrix $\beta$.

We fix the coordinate system by agreeing that the photon moves along the $z$-axis. This also introduces Bjorken's scaling variable,

$$
q_{0}=q_{3}-M_{N} x
$$

Furthermore we change the integration variable from $\vec{k}$ to $\vec{p}=\vec{q}+\vec{k}$. Taking into account the Jacobian for this transformation, which becomes feasible because only the regime $|\vec{p}| \approx 0$ contributes to the integral [3, 10], the hadronic tensor becomes

$$
\begin{aligned}
W_{\mu \nu}(q)=i \frac{N_{C}}{4} \int \frac{d \omega}{2 \pi} \sum_{\alpha} \int & d^{3} p\langle N| \\
+ & \left.\left.\tilde{\Psi}_{\alpha}^{\dagger}(\vec{p}) \mathcal{Q}_{A}^{2}\left(\tilde{\Psi}_{\alpha}^{\dagger}(\vec{p}) \mathcal{Q}_{A}^{2} \beta \gamma_{\nu}\right)_{5} \beta \gamma_{\mu} \not h \gamma_{\nu}(\vec{p}) f_{\alpha}^{-}(\omega)\right|_{\text {pole }}\right] \delta\left(p_{3}-M_{N} x+\omega\right) \\
& -\left[\left.\tilde{\Psi}_{\alpha}^{\dagger}(\vec{p}) \mathcal{Q}_{A}^{2} \gamma_{\nu} \not h \gamma_{\mu} \beta \tilde{\Psi}_{\alpha}(\vec{p}) f_{\alpha}^{+}(\omega)\right|_{\text {pole }}\right. \\
+ & \left.\left.\left.\tilde{\Psi}_{\alpha}^{\dagger}(\vec{p}) \mathcal{Q}_{A}^{2} \beta\left(\gamma_{\nu} \not h \gamma_{\mu}\right)_{5} \tilde{\Psi}_{\alpha}(\vec{p}) f_{\alpha}^{-}(\omega)\right|_{\text {pole }}\right] \delta\left(p_{3}-M_{N} x-\omega\right)\right\}|N\rangle .
\end{aligned}
$$

Here we have introduced the light-cone vector $\mathrm{n}^{\mu}=(1,0,0,1)^{\mu}$. Finally we adopt an exponential representation for the $\delta$-function which allows us to return to a coordinate space representation

$$
\begin{aligned}
W_{\mu \nu}(q)=-i M_{N} \frac{N_{C}}{4} \int \frac{d \omega}{2 \pi} \sum_{\alpha} \int d^{3} \xi \int \frac{d \lambda}{2 \pi} \mathrm{e}^{i M_{N} x \lambda} & \\
& \times\langle N|\left\{\left[\bar{\Psi}_{\alpha}(\vec{\xi}) \mathcal{Q}_{A}^{2} \gamma_{\mu} \not h \gamma_{\nu} \Psi_{\alpha}\left(\vec{\xi}+\lambda \hat{e}_{3}\right) \mathrm{e}^{-i \lambda \omega}\right.\right. \\
& \left.-\bar{\Psi}_{\alpha}(\vec{\xi}) \mathcal{Q}_{A}^{2} \gamma_{\nu} \not h \gamma_{\mu} \Psi_{\alpha}\left(\vec{\xi}-\lambda \hat{e}_{3}\right) \mathrm{e}^{i \lambda \omega}\right]\left.f_{\alpha}^{+}(\omega)\right|_{\text {pole }}
\end{aligned}
$$




$$
\begin{aligned}
& +\left[\bar{\Psi}_{\alpha}(\vec{\xi}) \mathcal{Q}_{A}^{2}\left(\gamma_{\mu} \not h \gamma_{\nu}\right)_{5} \Psi_{\alpha}\left(\vec{\xi}-\lambda \hat{e}_{3}\right) \mathrm{e}^{-i \lambda \omega}\right. \\
& \left.\left.\quad-\bar{\Psi}_{\alpha}(\vec{\xi}) \mathcal{Q}_{A}^{2}\left(\gamma_{\nu} \not h \gamma_{\mu}\right)_{5} \Psi_{\alpha}\left(\vec{\xi}+\lambda \hat{e}_{3}\right) \mathrm{e}^{i \lambda \omega}\right]\left.f_{\alpha}^{-}(\omega)\right|_{\text {pole }}\right\}|N\rangle,
\end{aligned}
$$

which is similar to the decomposition into quark and anti-quark distributions. Again, in the unregularized case, this simplifies enormously because $f_{\alpha}^{-}(\omega)=0$ while $\left.f_{\alpha}^{+}(\omega)\right|_{\text {pole }}=-4 \pi i \delta\left(\omega-\epsilon_{\alpha}\right)$. Apparently the hadronic tensor then indeed becomes a sum of quark and anti-quark distributions. However, in the Pauli-Villars regularized scheme, we have additional contributions from quark and anti-quark distributions with dispersion relations which also contain the cut-offs, $\Lambda_{i}$. Hence they differ from those dispersion relations naïvely expected from the solutions of the Dirac equation (2.15).

In the next step the hadronic tensor is contracted with appropriate projectors which in turn provides the structure functions. In the Bjorken limit these projectors become quite simple and are given in table 5.1. Note that within the Bjorken limit the Callan-Gross relation,

Table 5.1: Projection operators which extract the leading twist piece of the nucleon structure functions from the hadronic tensor. It should be remarked that the projectors given in the spin independent cases are not general but rather presume the contraction with $S_{\mu \nu \rho \sigma}$ which is defined before eq (4.7). The last row denotes the spin orientation of the nucleon.

\begin{tabular}{c|c|c|c}
$f_{1}$ & $f_{2}$ & $g_{1}$ & $g_{T}=g_{1}+g_{2}$ \\
\hline$-\frac{1}{2} g^{\mu \nu}$ & $-x g^{\mu \nu}$ & $\frac{i}{2 M_{N}} \epsilon^{\mu \nu \rho \sigma} \frac{q_{\rho} p_{\sigma}}{q \cdot s}$ & $\frac{-i}{2 M_{N}} \epsilon^{\mu \nu \rho \sigma} s_{\rho} p_{\sigma}$ \\
\hline spin & $\begin{array}{c}\text { spin } \\
\text { independent }\end{array}$ & $\vec{s} \| \vec{q}$ & $\vec{s} \perp \vec{q}$
\end{tabular}

$f_{2}(x)=2 x f_{1}(x)$, is automatically fulfilled. The unpolarized structure function $f_{1}(x)$ then becomes

$$
\begin{aligned}
f_{1}(x)=- & i M_{N} \frac{N_{C}}{2} \int \frac{d \omega}{2 \pi} \sum_{\alpha} \int d^{3} \xi \int \frac{d \lambda}{2 \pi} \mathrm{e}^{i M_{N} x \lambda}\left(\sum_{i=0}^{2} c_{i} \frac{\omega+\epsilon_{\alpha}}{\omega^{2}-\epsilon_{\alpha}^{2}-\Lambda_{i}^{2}+i \epsilon}\right)_{\text {pole }} \\
& \left.\times\langle N| \bar{\Psi}_{\alpha}(\vec{\xi}) \mathcal{Q}_{A}^{2} \not \not \Psi_{\alpha}\left(\vec{\xi}+\lambda \hat{e}_{3}\right) \mathrm{e}^{-i \omega \lambda}-\bar{\Psi}_{\alpha}(\vec{\xi}) \mathcal{Q}_{A}^{2} \not h \Psi_{\alpha}\left(\vec{\xi}-\lambda \hat{e}_{3}\right)\right) \mathrm{e}^{i \omega \lambda}|N\rangle \\
= & \left.i \frac{5}{36} M_{N} N_{C} \int \frac{d \omega}{2 \pi} \sum_{\alpha} \int \frac{d \lambda}{2 \pi} \mathrm{e}^{i M_{N} x \lambda}\left(\sum_{i=0}^{2} c_{i} \frac{\omega+\epsilon_{\alpha}}{\omega^{2}-\epsilon_{\alpha}^{2}-\Lambda_{i}^{2}+i \epsilon}\right)_{\text {pole }}\left(\vec{\xi}-\lambda \hat{e}_{3}\right) \mathrm{e}^{i \omega \lambda}\right\} \\
& \times \int d^{3} \xi\left\{\Psi_{\alpha}^{\dagger}(\vec{\xi})\left(1-\alpha_{3}\right) \Psi_{\alpha}\left(\vec{\xi}+\lambda \hat{e}_{3}\right) \mathrm{e}^{-i \omega \lambda}-\Psi_{\alpha}^{\dagger}(\vec{\xi})\left(1-\alpha_{3}\right) \Psi_{\alpha}(\vec{\xi})\right.
\end{aligned}
$$

to leading order in $1 / N_{C}$. Here we have employed parity invariance as well as the symmetry 
under grand-spin rotations to show that there is no iso-vector piecet. This is perfectly consistent with the Gottfried sum rule [34] vanishing at this order. Note that the spectral function in (5.16) is fully regularized. This indicates that this piece is associated to the real part of the bosonized action. In appendix D we also calculate the sub-leading order contribution to the unpolarized structure function $f_{1}(x)$, cf. eq (D.12) which expectedly is of iso-vector character. In contrast to the iso-scalar piece (5.16) that iso-vector contribution does not undergo regularization in the sense that the explicit dependence on the cut-off $\Lambda_{i}$ cancels. Of course, from our knowledge on the static property calculations in the NJL model it was to be expected that in case the iso-scalar piece is regularized the iso-vector piece is not and vice versa [9]. In particular, these findings show that the structure function which enters the Gottfried sum rule should not be regularized in contrast to previous studies. In ref [13, [1] this structure function has been treated analogously to the one of neutrino nucleon scattering associated with the Adler sum rule. As discussed in appendix D the latter indeed undergoes regularization. This example clearly exhibits that obtaining the formal expressions for structure functions from the defining action is unavoidable in cases when there is no relation to a static nucleon property.

Now we turn to the leading order in $1 / N_{C}$ piece to the polarized structure functions 5 . They are obtained from the anti-symmetric piece of the hadronic tensor

$$
\begin{aligned}
W_{\mu \nu}^{\mathrm{A}}=- & M_{N} \frac{N_{C}}{2} \epsilon_{\mu \rho \nu \sigma} \mathrm{n}^{\rho} \int \frac{d \omega}{2 \pi} \sum_{\alpha} \int d^{3} \xi \int \frac{d \lambda}{2 \pi} \mathrm{e}^{i M_{N} x \lambda}\left(\sum_{i=0}^{2} c_{i} \frac{\omega+\epsilon_{\alpha}}{\omega^{2}-\epsilon_{\alpha}^{2}-\Lambda_{i}^{2}+i \epsilon}\right)_{\text {pole }} \\
& \left.\times\langle N| \bar{\Psi}_{\alpha}(\vec{\xi}) \mathcal{Q}_{A}^{2} \gamma^{\sigma} \gamma_{5} \Psi_{\alpha}\left(\vec{\xi}+\lambda \hat{e}_{3}\right)\right) \mathrm{e}^{-i \omega \lambda}+\bar{\Psi}_{\alpha}(\vec{\xi}) \mathcal{Q}_{A}^{2} \gamma^{\sigma} \gamma_{5} \Psi_{\alpha}\left(\vec{\xi}-\lambda \hat{e}_{3}\right) \mathrm{e}^{i \omega \lambda}|N\rangle .
\end{aligned}
$$

Again, the spectral function is fully regularized as it originates from $f_{\alpha}^{+}(\omega)-f_{\alpha}^{-}(-\omega)$. Here the prescription (4.7) has a major impact. Without this specification the relative sign between the spectral functions would have been positive resulting in the spectral function $\left(\omega+\epsilon_{\alpha}\right) /\left(\omega^{2}-\right.$ $\left.\epsilon_{\alpha}^{2}+i \epsilon\right)$. In that case $W_{\mu \nu}^{\mathrm{A}}$ would have to be associated with unregularized imaginary part of the action. As we will see in the following section, such a result is not compatible with the sum rules. The reason is that the leading order (in $1 / N_{C}$ ) contributions to the axial charges stem from the regularized real part of the action. Using the relevant projection operator given in table 5.1 we find for the longitudinal polarized structure function

$$
\begin{aligned}
g_{1}(x) & =-i \frac{M_{N} N_{C}}{36}\left\langle N\left|I_{3}\right| N\right\rangle \int \frac{d \omega}{2 \pi} \sum_{\alpha} \int d^{3} \xi \int \frac{d \lambda}{2 \pi} \mathrm{e}^{i M_{N} x \lambda}\left(\sum_{i=0}^{2} c_{i} \frac{\omega+\epsilon_{\alpha}}{\omega^{2}-\epsilon_{\alpha}^{2}-\Lambda_{i}^{2}+i \epsilon}\right)_{\text {pole }} \\
\times & {\left[\Psi_{\alpha}^{\dagger}(\vec{\xi}) \tau_{3}\left(1-\alpha_{3}\right) \gamma_{5} \Psi_{\alpha}\left(\vec{\xi}+\lambda \hat{e}_{3}\right) \mathrm{e}^{-i \omega \lambda}+\Psi_{\alpha}^{\dagger}(\vec{\xi}) \tau_{3}\left(1-\alpha_{3}\right) \gamma_{5} \Psi_{\alpha}\left(\vec{\xi}-\lambda \hat{e}_{3}\right) \mathrm{e}^{i \omega \lambda}\right], }
\end{aligned}
$$

where we have substituted the matrix element (2.21) of the collective coordinates, $A$, sandwiched between nucleon states. Similarly the transverse combination $g_{T}(x)=g_{1}(x)+g_{2}(x)$ is

\footnotetext{
${ }^{2}$ Upon expanding in $\lambda$ the bilocal matrix element can be expressed as an infinite sum of local matrix elements. Due to parity invariance only matrix elements like

$$
\left(\lambda \partial_{3}\right)^{2 n}-\alpha_{3}\left(\lambda \partial_{3}\right)^{2 m+1}
$$

will be non-vanishing. As this operator is even under grand-spin reflections, the accompanying isospin operator must be so too.

${ }^{3}$ See ref [35] for an overview on spin dependent nucleon structure functions.
} 
given by

$$
\begin{gathered}
g_{T}(x)=-i \frac{M_{N} N_{C}}{36}\left\langle N\left|I_{3}\right| N\right\rangle \int \frac{d \omega}{2 \pi} \sum_{\alpha} \int d^{3} \xi \int \frac{d \lambda}{2 \pi} \mathrm{e}^{i M_{N} x \lambda}\left(\sum_{i=0}^{2} c_{i} \frac{\omega+\epsilon_{\alpha}}{\omega^{2}-\epsilon_{\alpha}^{2}-\Lambda_{i}^{2}+i \epsilon}\right)_{\text {pole }} \\
\times\left[\Psi_{\alpha}^{\dagger}(\vec{\xi}) \tau_{1} \alpha_{1} \gamma_{5} \Psi_{\alpha}\left(\vec{\xi}+\lambda \hat{e}_{3}\right) \mathrm{e}^{-i \omega \lambda}+\Psi_{\alpha}^{\dagger}(\vec{\xi}) \tau_{1} \alpha_{1} \gamma_{5} \Psi_{\alpha}\left(\vec{\xi}-\lambda \hat{e}_{3}\right) \mathrm{e}^{i \omega \lambda}\right] .
\end{gathered}
$$

In obtaining eqs $(5.18)$ and $(5.19)$ we have repeatedly made use of both parity invariance as well as the grand-spin reflection symmetry. As a result, the leading order pieces of the polarized structure functions are of iso-vector character.

We conclude this section by presenting an unpolarized 'structure function' which is not directly extracted from the Compton amplitude but nevertheless is of interest in the parton model picture. Reversing the relative sign of the contributions of the backward moving intermediate quarks, yields

$$
\begin{aligned}
\bar{f}_{1}(x)= & -M_{N} \frac{N_{C}}{2} \sum_{\alpha} \operatorname{sign}\left(\epsilon_{\alpha}\right) \int d^{3} \xi \int \frac{d \lambda}{2 \pi} \mathrm{e}^{i M_{N} x \lambda} \\
& \times\left\langle N\left|\bar{\Psi}_{\alpha}(\vec{\xi}) \mathcal{Q}_{A}^{2} \not h \Psi_{\alpha}\left(\vec{\xi}+\lambda \hat{e}_{3}\right) \mathrm{e}^{-i \epsilon_{\alpha} \lambda}+\bar{\Psi}_{\alpha}(\vec{\xi}) \mathcal{Q}_{A}^{2} \not h \Psi_{\alpha}\left(\vec{\xi}-\lambda \hat{e}_{3}\right) \mathrm{e}^{i \epsilon_{\alpha} \lambda}\right| N\right\rangle .
\end{aligned}
$$

This distribution could be thought of arising from coupling to an anti-hermitian flavor operator stemming from the exchange of a charged gauge boson. Another example for such a distribution emerges in neutrino-nucleon scattering which eventually leads to the Adler sum rule. In the unregularized version, the two different parts could be thought of being attributed to quark and anti-quark distributions. Therefore this 'structure function' will eventually be related to the baryon number distribution. In this piece the regularization has dropped out which made the extraction of the pole contribution simple. Using again the symmetries of the eigenfunctions of the Dirac Hamiltonian this 'structure function' turns out to be pure iso-scalar

$$
\begin{aligned}
\bar{f}_{1}(x)= & -M_{N} \frac{N_{C}}{2} \sum_{\alpha} \operatorname{sign}\left(\epsilon_{\alpha}\right) \int d^{3} \xi \int \frac{d \lambda}{2 \pi} \mathrm{e}^{i M_{N} x \lambda} \\
& \times\left[\Psi_{\alpha}^{\dagger}(\vec{\xi})\left(1-\alpha_{3}\right) \Psi_{\alpha}\left(\vec{\xi}+\lambda \hat{e}_{3}\right) \mathrm{e}^{-i \epsilon_{\alpha} \lambda}+\Psi_{\alpha}^{\dagger}(\vec{\xi})\left(1-\alpha_{3}\right) \Psi_{\alpha}\left(\vec{\xi}-\lambda \hat{e}_{3}\right) \mathrm{e}^{i \epsilon_{\alpha} \lambda}\right],
\end{aligned}
$$

where we have also omitted the factor associated with the charge matrix $\mathcal{Q}$.

\section{Sum rules}

Sum rules relate moments of the structure functions to static properties of hadrons and a consistently formulated model is required to satisfy these sum rules. Static properties are obtained by computing matrix elements of the symmetry currents. Here we want to reflect on the sum rules for the nucleon structure functions, in particular with regard to the prescription (4.7). As above, we will only consider the vacuum contribution because the one of the explicitly occupied valence level is not subject to regularization.

We recall that the symmetry currents are extracted from the terms linear in the external 
source contained in

$$
\begin{aligned}
\mathcal{A}_{\Lambda, \mathrm{R}}^{(1, v)}=-i \frac{N_{C}}{2} \sum_{i=0}^{2} c_{i} \operatorname{Tr}\{(- & \left.\mathbf{D}^{(\pi)} \mathbf{D}_{5}^{(\pi)}+\Lambda_{i}^{2}\right)^{-1} \\
& \left.\times\left[\psi T_{\mathrm{v}}^{a} \mathbf{D}_{5}^{(\pi)}-\mathbf{D}^{(\pi)} \psi T_{\mathrm{v}}^{a}+\not k \gamma_{5} T_{\mathrm{a}}^{a} \mathbf{D}_{5}^{(\pi)}+\mathbf{D}^{(\pi)} \not \nu \gamma_{5} T_{\mathrm{a}}^{a}\right]\right\}
\end{aligned}
$$

for the real part. Here $T_{\mathrm{v}, \mathrm{a}}^{a}$ denote appropriate flavor generators for the vector $(\mathrm{v})$ and axialvector (a) currents. Similarly the imaginary part of the action contributes

$$
\mathcal{A}_{\Lambda, \mathrm{I}}^{(1, v)}=-i \frac{N_{C}}{2} \operatorname{Tr}\left\{\left(-\mathbf{D}^{(\pi)} \mathbf{D}_{5}^{(\pi)}\right)^{-1}\left[\psi T_{\mathrm{v}}^{a} \mathbf{D}_{5}^{(\pi)}+\mathbf{D}^{(\pi)} \psi T_{\mathrm{v}}^{a}+\not l \gamma_{5} T_{\mathrm{a}}^{a} \mathbf{D}_{5}^{(\pi)}-\mathbf{D}^{(\pi)} \not l \gamma_{5} T_{\mathrm{a}}^{a}\right]\right\} .
$$

Again, we confine the present discussion to the leading order terms in the $1 / N_{C}$ expansion. The sub-leading order is relegated to appendix D. Reading off the coefficients of the source terms yields the charge operators

$$
Q_{\mu}^{a}=-i \frac{N_{C}}{2} D^{a b} \int \frac{d \omega}{2 \pi} \sum_{\alpha}\left\{\left\langle\alpha\left|T_{\mathrm{v}}^{b} \gamma_{\mu} \beta\right| \alpha\right\rangle f_{\alpha}^{-}(\omega)+\left\langle\alpha\left|\beta T_{\mathrm{v}}^{b} \gamma_{\mu}\right| \alpha\right\rangle f_{\alpha}^{+}(\omega)\right\}
$$

and

$$
Q_{\mu}^{(5) a}=i \frac{N_{C}}{2} D^{a b} \int \frac{d \omega}{2 \pi} \sum_{\alpha}\left\{\left\langle\alpha\left|T_{\mathrm{a}}^{b} \gamma_{\mu} \gamma_{5} \beta\right| \alpha\right\rangle f_{\alpha}^{-}(\omega)+\left\langle\alpha\left|\beta T_{\mathrm{a}}^{b} \gamma_{\mu} \gamma_{5}\right| \alpha\right\rangle f_{\alpha}^{+}(\omega)\right\} .
$$

Here we have introduced the adjoint representation of the collective coordinates for the soliton orientation, $D^{a b}=(1 / 2) \operatorname{tr}\left(A^{\dagger} \tau^{a} A \tau^{b}\right)$, which still has to be sandwiched between nucleon states, $c f$. eq (2.21). The similarity to the structure function expressions becomes apparent in the reappearance of the spectral functions (5.10). Adopting symmetric contours for the Cauchy integrals immediately yields the sum over all poles contained in the spectral functions $f_{\alpha}^{ \pm}$and the connection to the hadronic tensor $(5.12)$ is evident. Also note that in those matrix elements which are multiplied by $f_{\alpha}^{-}$, the Dirac matrix $\beta$ and the adjoint state $\langle\alpha|$ do not combine to $\bar{\Psi}_{\alpha}$.

The axial charges of the nucleon are obtained from the spatial components, $Q_{i}^{(5) a}$. While the iso-scalar part $\left(T_{\mathrm{a}}^{0}=1\right)$ vanishes at this order of the $1 / N_{C}$ expansion the iso-vector combination $\left(T_{\mathrm{a}}^{0}=\tau^{a} / 2\right)$ results in the vacuum contribution to the axial charge of the nucleon

$$
g_{\mathrm{A}}=\frac{N_{C}}{6}\left\langle N\left|2 I_{3}\right| N\right\rangle \sum_{i=0}^{2} c_{i} \sum_{\alpha} \frac{\epsilon_{\alpha}}{\sqrt{\epsilon_{\alpha}^{2}+\Lambda_{i}^{2}}}\left\langle\alpha\left|\tau_{3} \alpha_{3} \gamma_{5}\right| \alpha\right\rangle
$$

As remarked above, this quantity originates from the real part of the action as can be observed by the appearance of the Pauli-Villars regulator. In order to verify the sum rule for $g_{\mathrm{A}}$ the expression (6.5) has to be compared with the integral I $\int_{0}^{\infty} d x g_{1}(x)$, which is computed in several steps. We recognize that the two terms in square brackets in eq (5.18) are related by $\lambda \leftrightarrow-\lambda$

\footnotetext{
${ }^{1}$ The upper boundary being infinity is subject to the non-locality of the soliton. Eventually we have to boost the structure functions to the infinite momentum frame which properly projects them the interval $x \in[0,1], c f$. ref 36 .
} 
which allows us to extend the $x$-integration to minus infinity yielding $\left(2 \pi / M_{N}\right) \delta(\lambda)$ and whence a local matrix element. After performing the trivial $\lambda$-integration the odd powers of $\omega$ in the spectral function disappear

$$
\begin{aligned}
\int_{0}^{\infty} g_{1}(x)=i \frac{N_{C}}{36}\left\langle N\left|I_{3}\right| N\right\rangle \int \frac{d \omega}{2 \pi} \sum_{\alpha} & \left(\sum_{i=0}^{2} c_{i} \frac{\epsilon_{\alpha}}{\omega^{2}-\epsilon_{\alpha}^{2}-\Lambda_{i}^{2}+i \epsilon}\right)_{\text {pole }} \\
& \times \int d^{3} \xi \Psi_{\alpha}^{\dagger}(\vec{\xi}) \tau_{3} \alpha_{3} \gamma_{5} \Psi_{\alpha}(\vec{\xi}) .
\end{aligned}
$$

The quark matrix element of $\tau_{3} \gamma_{5}$ has vanished because of parity invariance. Also, the poles can easily be collected

$$
\left(\frac{1}{\omega^{2}-\epsilon_{\alpha}^{2}-\Lambda^{2}+i \epsilon}\right)_{\text {pole }}=-\frac{i \pi}{\sqrt{\epsilon_{\alpha}^{2}+\Lambda^{2}}}\left[\delta\left(\omega+\sqrt{\epsilon_{\alpha}^{2}+\Lambda^{2}}\right)+\delta\left(\omega-\sqrt{\epsilon_{\alpha}^{2}+\Lambda^{2}}\right)\right] .
$$

Hence the Bjorken sum rule

$$
\int_{0}^{\infty} d x\left(g_{1}^{\mathrm{p}}(x)-g_{1}^{\mathrm{n}}(x)\right)=\frac{1}{6} g_{\mathrm{A}}
$$

is straightforwardly obtained after taking care of the isospin matrix elements of the nucleon. Using rotational invariance in grand-spin space in addition to the above explained calculational rules verifies the Burkhardt-Cottingham [37] sum rule

$$
\int_{0}^{\infty} d x g_{2}(x)=0
$$

Here we wish to re-emphasize that the prescription (4.7) is crucial for deriving these results. Without it, the unregularized spectral function, $f^{+}(\omega)+f^{-}(-\omega)$, rather than $f^{+}(\omega)-f^{-}(-\omega)$ would have appeared in (6.6). The former would not have resulted in the regularized form for $g_{1}(x)$ as required by the Bjorken sum rule.

Although the treatment can be generalized to three flavors, we are currently focusing on two flavor case. Hence we require for the momentum sum rule

$$
\frac{9}{5} \int_{0}^{\infty} d x\left(f_{2}^{\mathrm{ep}}+f_{2}^{\mathrm{en}}\right)=\frac{36}{5} \int_{0}^{\infty} d x x f_{1}(x)
$$

according to the Callan-Gross relation and with $f_{1}(x)$ given in eq (5.16). The factor $x$ can be treated by differentiating with respect to $\lambda$. Furthermore the contribution from the backward propagating quarks serves to complete the $x$-integral along the negative half-line. That is,

$$
\begin{aligned}
\frac{36}{5} \int_{0}^{\infty} d x x f_{1}(x)= & -N_{C} \int \frac{d \omega}{2 \pi} \sum_{\alpha} \int \frac{d \lambda}{2 \pi} \int_{-\infty}^{\infty} d x \mathrm{e}^{i M_{N} x \lambda}\left(\sum_{i=0}^{2} c_{i} \frac{\omega+\epsilon_{\alpha}}{\omega^{2}-\epsilon_{\alpha}^{2}-\Lambda_{i}^{2}+i \epsilon}\right)_{\text {pole }} \\
& \times \frac{\partial}{\partial \lambda} \int d^{3} \xi \Psi_{\alpha}^{\dagger}(\vec{\xi})\left(1-\alpha_{3}\right) \Psi_{\alpha}\left(\vec{\xi}+\lambda \hat{e}_{3}\right) \mathrm{e}^{-i \omega \lambda} \\
= & -\frac{N_{C}}{M_{N}} \int \frac{d \omega}{2 \pi i} \sum_{\alpha}\left(\sum_{i=0}^{2} c_{i} \frac{\omega+\epsilon_{\alpha}}{\omega^{2}-\epsilon_{\alpha}^{2}-\Lambda_{i}^{2}+i \epsilon}\right)\left\langle\alpha\left|\left(1-\alpha_{3}\right)\left(i \partial_{3}+\omega\right)\right| \alpha\right\rangle .
\end{aligned}
$$


The subscript 'pole' has been dropped because without the oscillating function $\mathrm{e}^{ \pm i \omega \lambda}$ the spectral integral directly sums up the pole terms. The matrix elements of $i \partial_{3}$ and $\alpha_{3} \omega$ vanish due to parity invariance while the regularized sum of the matrix elements of $\alpha_{3} i \partial_{3}$ is zero for the field configuration which minimizes the soliton field energy ${ }^{2}[12]$. We are now left with

$$
\frac{36}{5} \int_{0}^{\infty} d x x f_{1}(x)=-\frac{N_{C}}{M_{N}} \int \frac{d \omega}{2 \pi i} \sum_{\alpha}\left(\sum_{i=0}^{2} c_{i} \frac{\omega^{2}}{\omega^{2}-\epsilon_{\alpha}^{2}-\Lambda_{i}^{2}+i \epsilon}\right) .
$$

Up to the factor $1 / M_{N}$ this can be shown to be the vacuum contribution to the soliton energy (2.17) when using the regularization condition $\sum_{i=0}^{2} c_{i}=0, c f$. eq (2.6). Hence we have verified the momentum sum rule. In particular we see that the model saturates this sum rule completely and there is no room for additional degrees of freedom, as e.g. gluons. In order to satisfy the momentum sum rule the iso-scalar content of $f_{1}$ apparently needs to be regularized. From the analogy to the computation of static properties we hence expect the iso-vector piece not to be regularized. This is, of course, perfectly consistent with our finding that the structure function entering the Gottfried sum rule, $f_{2}^{\mathrm{ep}}-f_{2}^{\mathrm{en}}=2 x\left(f_{2}^{\mathrm{ep}}-f_{2}^{\mathrm{en}}\right)$ remains unregularized as discussed in appendix D.

Above we have explicitly shown the necessity of the additional specification (4.7) to render the sum rules of the polarized structure functions consistent with the axial charges. On the formal level the above discussion is actually more transparent. When considering the Bjorken limit (4.6) and (4.8) we have already succeeded in expressing the two photon coupling in form of a linear coupling. What remains is the comparison of the structure of the Dirac matrices in $\psi(i \not \partial)^{-1} \psi$ and $\left(\psi(i \not \partial)^{-1} \psi\right)_{5}$ with that in the above expressions. Note that in eqs (4.6) and (4.8) we have retained the full dependence of the Dirac operator on the pion fields. In particular, for the soliton sector we have not yet carried out the expansion in the angular velocities (2.19), (5.4) and (5.5). According to eq (4.7) these contributions are given by

$$
\left(\psi(i \not)^{-1} \psi\right)_{(5)}=\mathcal{S} \cdot \mathcal{V} \pm \mathcal{E} \cdot \mathcal{A} \gamma_{5}
$$

Then the sum of (4.6) and (4.8) can be expressed as (up to the overall factor $N_{C} / 4 i$ )

$$
\begin{aligned}
& \mathcal{A}_{\Lambda}^{(2, v)}=\sum_{i=0}^{2} c_{i} \operatorname{Tr}\{(\left.-\mathbf{D}^{(\pi)} \mathbf{D}_{5}^{(\pi)}+\Lambda_{i}^{2}\right)^{-1} \\
&\left.\quad \times\left[\mathcal{Q}^{2}\left(\mathcal{S} \cdot \mathcal{V}+\mathcal{E} \cdot \mathcal{A} \gamma_{5}\right) \mathbf{D}_{5}^{(\pi)}-\mathbf{D}^{(\pi)}\left(\mathcal{S} \cdot \mathcal{V}-\mathcal{E} \cdot \mathcal{A} \gamma_{5}\right) \mathcal{Q}^{2}\right]\right\} \\
&+\operatorname{Tr}\left\{\left(-\mathbf{D}^{(\pi)} \mathbf{D}_{5}^{(\pi)}\right)^{-1}\left[\mathcal{Q}^{2}\left(\mathcal{S} \cdot \mathcal{V}+\mathcal{E} \cdot \mathcal{A} \gamma_{5}\right) \mathbf{D}_{5}^{(\pi)}+\mathbf{D}^{(\pi)}\left(\mathcal{S} \cdot \mathcal{V}-\mathcal{E} \cdot \mathcal{A} \gamma_{5}\right) \mathcal{Q}^{2}\right]\right\} \\
&=\sum_{i=0}^{2} c_{i} \operatorname{Tr}\left\{\left(-\mathbf{D}^{(\pi)} \mathbf{D}_{5}^{(\pi)}+\Lambda_{i}^{2}\right)^{-1}\right. \\
&\left.\quad \times\left[\mathcal{Q}^{2} \mathcal{S} \cdot \mathcal{V} \mathbf{D}_{5}^{(\pi)}-\mathbf{D}^{(\pi)} \mathcal{S} \cdot \mathcal{V} \mathcal{Q}^{2}+\mathcal{Q}^{2} \mathcal{E} \cdot \mathcal{A} \gamma_{5} \mathbf{D}_{5}^{(\pi)}+\mathbf{D}^{(\pi)} \mathcal{E} \cdot \mathcal{A} \gamma_{5} \mathcal{Q}^{2}\right]\right\}
\end{aligned}
$$

\footnotetext{
${ }^{2}$ We recall that we contribution of the valence quark orbit is always carried along.

${ }^{3}$ The additional factor ' $i$ ' is contained in the projectors which are listed in table 5.1.
} 


$$
\begin{aligned}
+\operatorname{Tr}\left\{\left(-\mathbf{D}^{(\pi)} \mathbf{D}_{5}^{(\pi)}\right)^{-1}\right. & \\
& \left.\times\left[\mathcal{Q}^{2} \mathcal{S} \cdot \mathcal{V} \mathbf{D}_{5}^{(\pi)}+\mathbf{D}^{(\pi)} \mathcal{S} \cdot \mathcal{V} \mathcal{Q}^{2}+\mathcal{Q}^{2} \mathcal{E} \cdot \mathcal{A} \gamma_{5} \mathbf{D}_{5}^{(\pi)}-\mathbf{D}^{(\pi)} \mathcal{E} \cdot \mathcal{A} \gamma_{5} \mathcal{Q}^{2}\right]\right\}
\end{aligned}
$$

This expression is exactly of the same structure as the sum of (6.1) and (6.2). It is this identical structure which renders the sum rules fulfilled. In case we had not introduced the relative sign in eq (4.7), which propagates through to eq (6.13), the last terms in the square brackets would have the opposite sign and would fail to match with the sum rules. When comparing eqs (6.1) and (6.2) to (6.14) we have not yet made explicit the 'position' of the $\beta$-matrices in $\mathbf{D}^{(\pi)}$ and $\mathbf{D}_{5}^{(\pi)}$ rather we have kept these operators in full. Thus this relative sign is not a matter of shuffling the Dirac matrix $\beta$.

We complete this section by noting that the sum rule for the 'structure function' $\bar{f}_{1}(x)$ indeed gives the baryon number of the polarized vacuum, i.e.

$$
\int_{0}^{\infty} d x \bar{f}_{1}(x)=-\frac{N_{C}}{2} \sum_{\alpha} \operatorname{sign}\left(\epsilon_{\alpha}\right) .
$$

\section{Conclusions}

In order to establish a self consistent regularization scheme for the hadron structure functions in the bosonized Nambu-Jona-Lasinio model we have studied the absorptive part of the Compton amplitude in Bjorken limit. Noting that the Compton amplitude is defined as the hadronic matrix element of a time-ordered correlation function of the symmetry currents, it is straightforwardly obtained from the regularized NJL action functional. In this respect, adopting the Compton amplitude as the preferred starting point for the structure function calculations yields self consistent results.

One constraint on regularizing the quark determinant is to maintain the anomaly structure of the quark loop when coupled to external sources. This is accomplished by regularizing only the $\gamma_{5}$ even piece (real part in Euclidean space) while leaving the conditionally convergent odd piece (imaginary part in Euclidean space) un-regularized. In a formal sense this leads to a model for scalar quarks which have gradient interactions thereby making the computation of the Compton amplitude more involved. Fortunately it turns out that in the Bjorken limit, which extracts the leading twist contribution of the structure functions, substantial simplifications occur. The reason is that the propagator, which carries the infinitely large momentum of the virtual photon in the quark loop, can be taken to be that of a free massless quark. Eventually this simplification also leads to the scaling laws of the structure functions. It is not apparent that these laws are maintained under regularization. In this respect we have seen that the Pauli-Villars regularization scheme for the bosonized NJL model is well suited because the cut-off's essentially act as masses which are additive to the momenta. For propagators which carry large external momenta the regularizing cut-off's can hence be ignored. Regularization schemes which exponentially suppress large momenta do not necessarily exhibit this feature. When studying the structure functions we have also recognized that treating the $\gamma_{5}$ even and 
odd pieces differently imposes further constraints on the regularization procedure in order to consistently formulate the sum rules. As this specification only effects the polarized structure functions its necessity has not been observed in previous calculations of the pion structure functions. Here it is worth noting that a dissimilar regularization of the $\gamma_{5}$ even and odd pieces requires a careful treatment in other cases as well. One example is the inclusion of $\omega$ vector meson in the NJL model [38, 39].

Having made this specification the calculation of nucleon structure functions from the Compton amplitude in the NJL chiral soliton model turned out to be sensible. The sum rules for the fully regularized structure functions are straightforwardly verified because Cutkosky's rules, which define the absorptive part of the amplitude, extract exactly the same poles from the spectral integral as do the Cauchy integrals for the matrix elements associated with static nucleon properties. We have found that in general the structure function cannot be written as linear combinations of quark and anti-quark distributions; rather they acquire contributions from various poles in the quark propagators which are associated with the Pauli-Villars cut-off's as the latter play the role of quark masses. Only those combinations of structure functions which are related to the $\gamma_{5}$ odd piece and hence are not regularized turn out to be simple sums of quark and ant-quark distributions. When including cranking corrections to generate nucleon quantum numbers from the chiral soliton we have seen that only either the iso-scalar or the iso-vector part of the considered structure function undergoes regularization. Of course, this is expected from the computation of nucleon charges in this model. Surprisingly, in the case of the structure function which enters the Gottfried sum rule we have seen that it is the iso-scalar rather than the iso-vector part which undergoes regularization. This has not been expected as this iso-vector piece is very similar to the structure function entering the Adler sum rule for (anti) neutrino nucleon scattering, which indeed gets regularized.

Certainly this study represents the first step towards a full self-consistent calculation which will in turn require numerical analyses. We have already noted that in the NJL model many of the nucleon properties are dominated by the corresponding contribution of the distinct valence level which does not undergo regularization. Hence we do not expect drastic changes in comparison with previous calculations. Nevertheless, in light of the fact that the iso-vector component of the unpolarized structure function $f_{1}$ remains unregularized suggests a thorough re-evaluation of the Gottfried sum rule is necessary. In addition, in the case of structure functions which are subject to regularization, and hence are more difficult to access, one might eventually confine oneself to the evaluation of low moments being related to products of quark matrix elements of local operators. As an example we will consider $d_{2}$, the second moment of the twist- 3 piece of the transverse polarized structure function, $g_{2}(x)$ which currently is of experimental interest [40.

\section{Acknowledgments}

We are grateful to $\mathrm{H}$. Reinhardt for the discussions and perspective during the many phases of this project. In addition H.W. would like to thank R. L. Jaffe for interesting discussions. L.G. wishes to thank K. A. Milton for support on this project. 


\section{Appendix A: Pion structure function from the Bethe-Salpeter amplitude}

In ref [17 and section 3 the pion structure functions were obtained from analyzing the Compton amplitude. In this appendix we will show that these structure functions can directly be obtained from the pion Bethe-Salpeter amplitude. This is completely consistent with the regularization and does not require to introduce any transverse cut-off. As we will see this can be done in terms of light-cone wave-functions handled in a proper way. To this end we consider the $\gamma_{5}$-even Pauli-Villars regularized effective action, up to second order in the pion field. This yields the correlation function in terms of the Bethe-Salpeter bound state amplitude $\chi(p, k)$ defined by

$$
\left.\mathcal{A}_{N J L}\right|_{\pi \pi}=\frac{1}{2} \int \frac{d^{4} p}{(2 \pi)^{4}} \int \frac{d^{4} k}{(2 \pi)^{4}} \overrightarrow{\tilde{\pi}}(p) \cdot \overrightarrow{\tilde{\pi}}(-p)\left(p^{2}-m_{\pi}^{2}\right) \chi(p, k) .
$$

According to eqs (2.10) and (2.12) the Bethe-Salpeter amplitude in the Pauli-Villars regularization scheme is

$$
\chi(p, k)=\left.4 N_{c} \mathrm{i} g^{2} \frac{d}{d p^{2}}\left\{p^{2} \sum_{i} c_{i} \frac{1}{k^{2}-m^{2}-\Lambda_{i}^{2}+\mathrm{i} \epsilon} \frac{1}{(k-p)^{2}-m^{2}-\Lambda_{i}^{2}+\mathrm{i} \epsilon}\right\}\right|_{p^{2}=m_{\pi}^{2}} .
$$

Let us introduce light-cone (LC) variables

$$
k^{+}=k^{0}+k^{3}, \quad k^{-}=k^{0}-k^{3}, \quad d^{4} k=\frac{1}{2} d k^{+} d k^{-} d^{2} k_{\perp} .
$$

Employing the definition"

$$
\Psi_{\pi}^{2}\left(x, k_{\perp}\right)=\left.\int d k^{-} \chi(p, k)\right|_{k^{+}=m_{\pi} x, p^{2}=m_{\pi}}
$$

for the LC wave-function we find

$$
\begin{aligned}
\Psi_{\pi}^{2}\left(x, k_{\perp}\right)=\frac{4 N_{c} \mathrm{i} g^{2}}{x(1-x)} & \frac{d}{d m_{\pi}^{2}}\left\{m_{\pi}^{2} \int \frac{d k^{-}}{2} \frac{m_{\pi} x}{k^{-} m_{\pi} x-k_{\perp}^{2}+m^{2}+\Lambda_{i}^{2}-\mathrm{i} \epsilon}\right. \\
& \left.\times \frac{1}{k^{-} m_{\pi}(x-1)-m_{\pi}^{2}(x-1)-k_{\perp}^{2}+m^{2}+\Lambda_{i}^{2}-\mathrm{i}}\right\} .
\end{aligned}
$$

The poles in the complex $k^{-}$plane are located at

$$
k^{-}=\frac{m^{2}+k_{\perp}^{2}+\Lambda_{i}^{2}-\mathrm{i} \epsilon}{m_{\pi} x} \text { and } \quad k^{-}=m_{\pi}+\frac{m^{2}+k_{\perp}^{2}+\Lambda_{i}^{2}-\mathrm{i} \epsilon}{m_{\pi}(x-1)} .
$$

For $x>1$ or $x<0$ both poles are above and below the real axis respectively, and hence the integral vanishes in either case. For $0<x<1$ the integral yields the LC wave-function

$$
\Psi_{\pi}^{2}\left(x, k_{\perp}\right)=\frac{4 N_{c} g^{2}}{(2 \pi)^{2}} \pi \frac{d}{d m_{\pi}^{2}}\left\{m_{\pi}^{2} \sum_{i} c_{i} \frac{1}{k_{\perp}^{2}+m^{2}+\Lambda_{i}^{2}-m_{\pi}^{2} x(1-x)-\mathrm{i} \epsilon}\right\} .
$$

\footnotetext{
${ }^{1}$ We could equivalently define the equal time wave-function $\Psi_{\pi}^{2}(\vec{k})=\left.\int d k_{0} \chi(p, k)\right|_{p^{2}=m_{\pi}^{2}}$ and go to the infinite momentum frame, $p^{0} \rightarrow P+m_{\pi}^{2} /(2 P), p^{3} \rightarrow P$ and $p_{\perp}=0_{\perp}, k^{3}=x P$.
} 
Notice that, due to the Pauli-Villars subtractions, we have the asymptotic behavior in the transverse momentum $k_{\perp}$,

$$
\Psi_{\pi}\left(x, k_{\perp}\right)^{2} \sim \frac{4 N_{c} g^{2}}{(2 \pi)^{2}} \pi \frac{\sum_{i} c_{i} \Lambda_{i}^{4}}{k_{\perp}^{6}} .
$$

This guarantees the convergence of the $k_{\perp}$ integral without introducing a transverse cut-off. Thus, the Pauli-Villars regulators automatically provide a form of a transverse cut-off. Upon integrating the transverse momentum we get the structure function

$$
F(x)=\int \frac{d^{2} k_{\perp}}{(2 \pi)^{2}}\left\{\left(\frac{1}{3}\right)^{2} \Psi_{\pi, d}^{2}\left(x, k_{\perp}\right)+\left(\frac{2}{3}\right)^{2} \Psi_{\pi, u}^{2}\left(x, k_{\perp}\right)\right\},
$$

which coincides with the result found in ref [17] and eq (3.7) of section 3. The light-cone interpretation has been pursued before in the unregularized case (see e.g. [31] and refs therein) and more recently [41, 42] within a LC quantization. In these cases transverse cut-off's were introduced, a posteriori. As we have shown above this is not necessary. Also, the LC wavefunction approach is a bit subtle in the present context due to the necessary regularization. For instance, the well known LC convolution formulas for the elastic pion form factor 43], are only valid here in conjunction with the regularization. That is, by writing the LC wave-function as a Pauli-Villars sum

$$
\Psi_{\pi}^{2}\left(x, k_{\perp}\right)=\sum_{i} c_{i} \Psi_{\pi, i}^{2}\left(x, k_{\perp}\right)
$$

we have the regularized null-plane formula, $F\left(q^{2}\right)=\sum_{i} c_{i} F_{i}\left(q^{2}\right)$ for the elastic pion form factor. This is not the same as computing $\Psi\left(x, k_{\perp}\right)$ and employing the convolution formula.

\section{Appendix B: Separation into free-massless and full quark propagators}

In this appendix we reinforce the analysis presented in section 4 . There we argued that in the Bjorken limit one of the two propagators which are involved in the calculation of the structure functions can be reduced to the free massless one. Here we do this by means of a symmetric version of the Wigner transformation (WT) as defined in ref 44. Although this technique or similar ones are well known in other fields, it is perhaps not very familiar in the present context. Because of that and in order to set up the notation we will review it briefly. The main advantage of using this method lies in the possibility of a systematic evaluation of higher twist contributions to structure functions in the soliton model.

Using translational invariance and localized baryon states $|B\rangle$ with normalization $\langle B \mid B\rangle=$ 1 , one has the convenient formula for the Compton amplitude of virtual photons in the rest frame of the soliton target

$$
(-i) T_{\mu \nu}(p, q)=2 M_{N} \int d^{3} R \int d^{4} \xi e^{i q \cdot \xi}\left\langle B\left|T\left\{J_{\mu}\left(R+\frac{\xi}{2}\right) J_{\nu}\left(R-\frac{\xi}{2}\right)\right\}\right| B\right\rangle,
$$

where, as usual, $J_{\mu}(x)=\bar{q}(x) \mathcal{Q} \gamma_{\mu} q(x)$. This equation is equivalent to eq (5.1) if translational invariance is used. The form (B.1), however, is slightly more convenient to prove the announced 
result. As discussed in section 5 the vacuum contribution can be obtained by a suitable functional differentiation of the fermion determinant with respect to the external sources. In the unregularized case we get

$$
\left.T_{\mu \nu}(p, q)\right|_{\mathrm{vac}}=-M_{N} N_{c} \int d^{3} R \int d^{4} \xi e^{\mathrm{i} q \cdot \xi} \operatorname{tr}\left(\gamma_{\mu} \mathcal{Q}\left\langle R+\frac{\xi}{2}|\mathbf{S}| R-\frac{\xi}{2}\right\rangle \gamma_{\nu} \mathcal{Q}\left\langle R-\frac{\xi}{2}|\mathbf{S}| R+\frac{\xi}{2}\right\rangle\right)+\text { cr. }
$$

where the propagator operator is defined as $\mathbf{S}:=(\mathrm{iD})^{-1}$, the trace is taken with respect to Dirac and flavor degrees of freedom while "cr." denotes the crossed contribution $(q \rightarrow-q, \mu \rightarrow \nu)$, i.e. backward moving quarks. Schematically we have expressions of the form

$$
\int d^{4} \xi e^{\mathrm{i} q \cdot \xi}\left\langle x+\frac{\xi}{2}|A| x-\frac{\xi}{2}\right\rangle\left\langle x-\frac{\xi}{2}|B| x+\frac{\xi}{2}\right\rangle,
$$

i.e., the Fourier transformation of a product of bilocal functions. To deal with these expressions properly we use the following symmetric definition of the WT as considered in ref [44]. For any bilocal operator $A$, with matrix elements $\left\langle x|A| x^{\prime}\right\rangle$ let us define its symmetric Wigner representation as

$$
A(x, p)=\int \mathrm{d}^{4} \xi e^{i \xi p}\left\langle x+\frac{\xi}{2}|A| x-\frac{\xi}{2}\right\rangle
$$

Using its inverse,

$$
\left\langle x+\frac{\xi}{2}|A| x-\frac{\xi}{2}\right\rangle=\int \frac{d^{4} p}{(2 \pi)^{4}} e^{-\mathrm{i} \xi \cdot p} A(x, p),
$$

we get

$$
\int d^{4} \xi e^{\mathrm{i} q \cdot \xi}\left\langle x+\frac{\xi}{2}|A| x-\frac{\xi}{2}\right\rangle\left\langle x-\frac{\xi}{2}|B| x+\frac{\xi}{2}\right\rangle=\int \frac{d^{4} p}{(2 \pi)^{4}} A(x, p) B(x, p-q) .
$$

Notice that we have arbitrarily chosen the operator $B$ to carry the photon momentum. The product of two operators satisfies the following expression for its WT

$$
\begin{aligned}
(A B)(x, p) & =e^{\frac{\mathrm{i}}{2}\left(\partial_{p}^{A} \cdot \partial_{x}^{B}-\partial_{p}^{B} \cdot \partial_{x}^{A}\right)} A(x, p) B(x, p) \\
& =A\left(x-\frac{\mathrm{i}}{2} \partial_{p}, p+\frac{\mathrm{i}}{2} \partial_{x}\right) B(x, p) \\
& =\sum_{n=0}^{\infty} \sum_{m=0}^{\infty} \frac{i^{n}}{2^{n} n !} \frac{(-i)^{m}}{2^{m} m !} \frac{\partial^{n+m} A(x, p)}{\partial p_{\nu_{1}} \cdots \partial p_{\nu_{n}} \partial x_{\mu_{1}} \cdots \partial x_{\mu_{m}}} \frac{\partial^{n+m} B(x, p)}{\partial x^{\nu_{1}} \cdots \partial x^{\nu_{n}} \partial p^{\mu_{1}} \cdots \partial p^{\mu_{m}}} .
\end{aligned}
$$

In the limit of large momentum $p$ one clearly gets

$$
(A B)(x, p) \rightarrow A\left(x-\frac{\mathrm{i}}{2} \partial_{p}, p\right) B(x, p) .
$$

The WT of the Dirac operators $\mathbf{D}$ and $\mathbf{D}_{5}$ are

$$
\mathrm{iD}(x, p)=\not p-\mathcal{M}(x) \quad \text { and } \quad \mathrm{iD}_{5}(x, p)=-\not p-\mathcal{M}_{5}(x),
$$

respectively. Applying the product rule, eq.(B.7) to the operator identity iDS $=1$ we obtain

$$
\mathrm{i} \mathbf{D}\left(x-\frac{\mathrm{i}}{2} \partial_{p}, p+\frac{\mathrm{i}}{2} \partial_{x}\right) \mathbf{S}(x, p)=1 .
$$


Hence the WT of the propagator is given by

$$
\mathbf{S}(x, p)=\frac{1}{\not p+\frac{\mathrm{i}}{2} \not_{x}-\mathcal{M}\left(x-\frac{\mathrm{i}}{2} \partial_{p}\right)} .
$$

This expression is suitable for a derivative expansion in the field $\mathcal{M}(x)$ as follows

$$
\mathbf{S}(x, p)=\mathbf{S}_{0}(x, p)+\mathbf{S}_{1}(x, p)+\mathbf{S}_{2}(x, p)+\ldots,
$$

where the subscript indicates the number of derivatives in $\mathcal{M}(x)$. The derivatives act to the right. Therefore the lowest orders in this derivative expansions are given by

$$
\begin{aligned}
\mathbf{S}_{0}(x, p) & =\frac{1}{\not p-\mathcal{M}(x)} \\
\mathbf{S}_{1}(x, p) & =\frac{\mathrm{i}}{2} \mathbf{S}_{0}(x, p)\left(\not \partial_{x}-\partial_{x} \mathcal{M}(x) \cdot \partial_{p}\right) \mathbf{S}_{0}(x, p)
\end{aligned}
$$

The proper way to evaluate first and higher order expressions is by commuting the derivatives to the right until they annihilate the unit operator. For instance, in the first order of derivatives we have

$$
\mathbf{S}_{1}(x, p)=\frac{\mathrm{i}}{2} \mathbf{S}_{0}(x, p)\left(\gamma_{\mu} \mathbf{S}_{0}(x, p) \partial^{\mu} \mathcal{M}(x)+\partial^{\mu} \mathcal{M}(x) \mathbf{S}_{0}(x, p) \gamma_{\mu}\right) \mathbf{S}_{0}(x, p) .
$$

As we see the number of zeroth order propagators increases due to the coordinate and momentum derivatives. It actually is this expansion which is indicated in figure 4.2. Now it is obvious that in the limit of large momentum $p$ higher order terms in $\mathbf{S}_{n}(x, p)$ can be neglected as compared to the zeroth order one, $\mathbf{S}_{0}(x, p)$. Finally the latter can be replaced by the free massless one, $\mathbf{S}_{F}(x, p)$. In summary,

$$
\mathbf{S}(x, p) \rightarrow \mathbf{S}_{F}(x, p)=\frac{1}{\not p} .
$$

Notice that in the expression (B.6) only one operator contains the momentum $q$. Therefore, we finally get in the limit of large momentum $q$,

$$
\begin{aligned}
& \left.T_{\mu \nu}(p, q)\right|_{\text {sea }}=N_{c} \int d^{3} R \int \frac{d^{4} p}{(2 \pi)^{4}} \operatorname{tr}\left(\gamma_{\mu} \mathcal{Q} \mathbf{S}(R, p) \gamma_{\nu} \mathcal{Q} \mathbf{S}(R, p-q)\right)+\mathrm{cr} . \\
& \rightarrow N_{c} \int d^{3} R \int d^{4} \xi e^{\mathrm{i} q \cdot \xi} \operatorname{tr}\left(\gamma_{\mu} \mathcal{Q}\left\langle R+\frac{\xi}{2}|\mathbf{S}| R-\frac{\xi}{2}\right\rangle \gamma_{\nu} \mathcal{Q}\left\langle-\frac{\xi}{2}\left|\mathbf{S}_{F}\right| \frac{\xi}{2}\right\rangle\right)+\mathrm{cr} .
\end{aligned}
$$

where we have already used that the free propagator is invariant under translations. Thus, in the Bjorken limit we may replace that Dirac operator through which the large photon momentum flows by the free one. Also note that in section 4 we have made this replacement before taking the functional derivatives with respect to the vector sources. In the present context, this

\footnotetext{
${ }^{1}$ This is effectively a semiclassical expansion of the propagator as can be seen by reinserting $\hbar$ in the previous expressions $\partial_{x} \rightarrow \hbar \partial_{x}$ and $\partial_{p} \rightarrow \hbar \partial_{p}$
} 
corresponds to summing over the two possibilities that either of the two propagators carries the large momentum.

In the regularized case the argument is a bit more involved but the result is similar. The only point is to identify the operators $A$ and $B$. Using eq (B.7), we get

$$
\begin{aligned}
-\mathbf{D D}_{5}(x, p) & =-\left(\not p+\frac{\mathrm{i}}{2} \not_{x}-\mathcal{M}\left(x-\frac{\mathrm{i}}{2} \partial_{p}\right)\right)\left(\not p+\mathcal{M}_{5}(x)\right) \\
& =-(\not p-\mathcal{M}(x))\left(\not p+\mathcal{M}_{5}(x)\right)+\mathrm{i} \not_{x} \mathcal{M}_{5}(x)
\end{aligned}
$$

where in the second line we have used that $\mathcal{M} \gamma_{\mu}=\gamma_{\mu} \mathcal{M}_{5}$. Several cases for $A$ and $B$ have to be considered. From the asymptotic limit of the product rule one deduces that the WT of a derivative operator of a given order is a polynomial in $p$ of the same order. In the limit of large momentum this polynomial dominates over the potential piece. Due to this reason one has the simplification $\mathbf{D D}_{5}+\Lambda_{i}^{2} \rightarrow \mathbf{D D}_{5}$ in the large momentum limit. Furthermore,

$$
i \mathbf{D}_{5}\left(-\mathbf{D D}_{5}+\Lambda_{i}^{2}\right)^{-1} \rightarrow(i \mathbf{D})^{-1} \quad \text { and } \quad\left(-\mathbf{D D}_{5}+\Lambda_{i}^{2}\right)^{-1} i \mathbf{D} \rightarrow\left(i \mathbf{D}_{5}\right)^{-1}
$$

In summary, the simplification given by eq.4.6) is supported by this analysis. Moreover, it makes evident that the Pauli-Villars regularization leads to the scaling behavior. This is not apparent in other schemes which do not have the regulating cut-off in the polynomial piece.

\section{Appendix C: Sum rules in the $D_{5}$ model}

In this appendix we illustrate that the unphysical nature of the $\mathbf{D}_{5}$ model is reflected by the sum rules for the polarized structure functions. This discussion will take place on the formal level and whence we ignore regularization. According to eq (1.2) the $\mathbf{D}_{5}$ model is defined as

$$
\mathcal{A}_{5}=-i N_{C} \operatorname{Tr} \log \left\{i \mathbf{D}_{5}\right\}
$$

The static properties are obtained from

$$
\begin{aligned}
& \mathcal{A}^{(1, v)}=i N_{C} \operatorname{Tr}\left\{\left(i \mathbf{D}^{(\pi)}\right)^{-1}\left[\psi T_{\mathrm{v}}^{a}+\not h \gamma_{5} T_{\mathrm{a}}^{a}\right]\right\}, \\
& \mathcal{A}_{5}^{(1, v)}=-i N_{C} \operatorname{Tr}\left\{\left(i \mathbf{D}_{5}^{(\pi)}\right)^{-1}\left[\psi T_{\mathrm{v}}^{a}-\not \phi \gamma_{5} T_{\mathrm{a}}^{a}\right]\right\}
\end{aligned}
$$

within the physical $\mathbf{D}$ and unphysical $\mathbf{D}_{5}$ models, respectively. Omitting, for the time being, the collective coordinates, the charges are given by

$$
Q_{\mu}^{a}=\sum_{\alpha} \operatorname{sign}\left(\epsilon_{\alpha}\right)\left\langle\alpha\left|\beta \gamma_{\mu}\left(\gamma_{5}\right) T_{\mathrm{v}, \mathrm{a}}^{a}\right| \alpha\right\rangle \quad \text { and } \quad Q_{5, \mu}^{a}=-\sum_{\alpha} \operatorname{sign}\left(\epsilon_{\alpha}\right)\left\langle\alpha\left|\gamma_{\mu}\left(-\gamma_{5}\right) \beta T_{\mathrm{v}, \mathrm{a}}^{a}\right| \alpha\right\rangle
$$

For convenience, we have put vector and axial-vector generators together. To study the sum rules we require the hadronic tensor, $W_{\mu \nu ; 5}$ within the $\mathbf{D}_{5}$ model. In analogy to eq (4.9), $W_{\mu \nu ; 5}$ will be generated by

$$
\mathcal{A}_{5}^{(2, v)}=i \frac{N_{C}}{2} \operatorname{Tr}\left\{\left(\mathbf{D}_{5}^{(\pi)}\right)^{-1}\left[\mathcal{Q}^{2} \psi(-\not \partial)^{-1} \psi\right]\right\} .
$$


It should be recalled that $(-i \not \partial)^{-1}$ represents the free massless quark propagator in the $\mathbf{D}_{5}$ model, see eq (2.2). As can directly be observed, the difference among the pole contributions between the $\mathbf{D}$ and $\mathbf{D}_{5}$ models

$$
\left(\frac{ \pm \omega+\epsilon_{\alpha}}{\omega^{2}-\epsilon_{\alpha}^{2}+i \epsilon}\right)_{\text {pole }}=2 \pi i\left( \pm \omega+\epsilon_{\alpha}\right) \delta\left(\omega^{2}-\epsilon_{\alpha}^{2}\right)=2 \pi i \operatorname{sign}\left(\epsilon_{\alpha}\right)
$$

has no effect. For the same reason the relative overall signs did not change when going from eq (C.2) to eq (C.3). Besides the overall sign the only difference between $\mathcal{A}_{5}^{(2, v)}$ and (4.9) is therefore the position of the Dirac matrix $\beta$ contained in $\mathbf{D}_{5}^{(\pi)}$,

$$
\begin{aligned}
W_{\mu \nu ; 5}=- & M_{N} N_{C} \sum_{\alpha} \operatorname{sign}\left(\epsilon_{\alpha}\right) \int d t \int d^{3} \xi_{1} \int d^{3} \xi_{2} \int \frac{d^{4} k}{(2 \pi)^{4}} \mathrm{e}^{i t\left(q^{0}+k^{0}\right)} \mathrm{e}^{-i\left(\vec{\xi}_{1}-\vec{\xi}_{2}\right) \cdot(\vec{q}+\vec{k})} \delta\left(k^{2}\right) \\
& \times\left\langle N\left|\left\{\Psi_{\alpha}^{\dagger}\left(\vec{\xi}_{1}\right) \mathcal{Q}_{A}^{2} \gamma_{\mu} \not k \gamma_{\nu} \beta \Psi_{\alpha}\left(\vec{\xi}_{2}\right) \mathrm{e}^{i \epsilon_{\alpha} t}-\Psi_{\alpha}^{\dagger}\left(\vec{\xi}_{2}\right) \mathcal{Q}_{A}^{2} \gamma_{\nu} \not k \gamma_{\mu} \beta \Psi_{\alpha}\left(\vec{\xi}_{1}\right) \mathrm{e}^{-i \epsilon_{\alpha} t}\right\}\right| N\right\rangle .
\end{aligned}
$$

In comparison with the expressions (C.3) this particular position of $\beta$ actually is a desired result. When reducing the product of Dirac matrices

$$
\begin{aligned}
W_{\mu \nu ; 5}=- & M_{N} N_{C} \sum_{\alpha} \operatorname{sign}\left(\epsilon_{\alpha}\right) \int d t \int d^{3} \xi_{1} \int d^{3} \xi_{2} \int \frac{d^{4} k}{(2 \pi)^{4}} \mathrm{e}^{i t\left(q^{0}+k^{0}\right)} \mathrm{e}^{-i\left(\vec{\xi}_{1}-\vec{\xi}_{2}\right) \cdot(\vec{q}+\vec{k})} \delta\left(k^{2}\right) k^{\sigma} \\
& \times\langle N|\left\{S_{\mu \rho \nu \sigma}\left[\Psi_{\alpha}^{\dagger}\left(\vec{\xi}_{1}\right) \mathcal{Q}_{A}^{2} \gamma^{\rho} \beta \Psi_{\alpha}\left(\vec{\xi}_{2}\right) \mathrm{e}^{i \epsilon_{\alpha} t}-\Psi_{\alpha}^{\dagger}\left(\vec{\xi}_{2}\right) \mathcal{Q}_{A}^{2} \gamma^{\rho} \beta \Psi_{\alpha}\left(\vec{\xi}_{1}\right) \mathrm{e}^{-i \epsilon_{\alpha} t}\right]\right. \\
& \left.-i \epsilon_{\mu \rho \nu \sigma}\left[\Psi_{\alpha}^{\dagger}\left(\vec{\xi}_{1}\right) \mathcal{Q}_{A}^{2} \gamma^{\rho} \gamma_{5} \beta \Psi_{\alpha}\left(\vec{\xi}_{2}\right) \mathrm{e}^{i \epsilon_{\alpha} t}+\Psi_{\alpha}^{\dagger}\left(\vec{\xi}_{2}\right) \mathcal{Q}_{A}^{2} \gamma^{\rho} \gamma_{5} \beta \Psi_{\alpha}\left(\vec{\xi}_{1}\right) \mathrm{e}^{-i \epsilon_{\alpha} t}\right]\right\}|N\rangle
\end{aligned}
$$

we recognize that in comparison with the hadronic tensor in the physical model (5.6) there appears no new relative sign between vector and axial-vector pieces. However, such a sign would be demanded to comply with the sum rules of (C.3) which indeed has a relative sign between the vector and axial-vector pieces. We conclude that a world defined by $\mathbf{D}_{5}$ the Bjorken sum rule would apparently read

$$
\int d x\left(g_{1}^{\mathrm{p}}(x)-g_{1}^{\mathrm{n}}(x)\right)=-\frac{1}{6} g_{\mathrm{A}} .
$$

This, of course, would be a major inconsistency which we have resolved by the prescription (4.7).

\section{Appendix D: Cranking corrections to structure functions}

As for the leading order in the $1 / N_{C}$ expansion, we confine ourselves to the vacuum contribution because the piece which is due to the explicit occupation of the valence level has already been discussed previously [10].

The computation of the cranking corrections to the nucleon structure functions starts with expanding the expressions (4.6) and (4.8) to linear order in the angular velocities $\vec{\Omega}$, which are defined in eq (2.19). In doing so, we will repeatedly use the relations (5.4) and (5.5). 
To linear order in $\vec{\Omega}$ we then find that part of the action which is quadratic in the vector sources $v_{\mu}$ to be

$$
\begin{aligned}
& \mathcal{A}^{(2)}=-i \frac{N_{C}}{4} \int \frac{d \omega}{2 \pi} \sum_{\alpha}\left\{\left\langle\omega, \alpha\left|\mathcal{Q}_{A}^{2} \beta \psi(i \not \partial)^{-1} \psi\right| \omega, \alpha\right\rangle f_{\alpha}^{+}(\omega)\right. \\
& +\left\langle\omega, \alpha\left|\mathcal{Q}_{A}^{2}\left[\psi(i \not \partial)^{-1} \psi\right]_{5} \beta\right| \omega, \alpha\right\rangle f_{\alpha}^{-}(\omega) \\
& +\frac{i}{2}\left\langle\omega, \alpha\left|\beta\left[\psi(i \not \partial)^{-1} \psi \hat{t} \mathcal{Q}_{A}^{2} \vec{\tau} \cdot \vec{\Omega}-\vec{\tau} \cdot \vec{\Omega} \mathcal{Q}_{A}^{2} \hat{t} \psi(i \not \partial)^{-1} \psi\right]\right| \omega, \alpha\right\rangle f_{\alpha}^{+}(\omega) \\
& +\frac{i}{2}\left\langle\omega, \alpha\left|\left[\psi(i \not \partial)^{-1} \psi \hat{t} \mathcal{Q}_{A}^{2} \vec{\tau} \cdot \vec{\Omega}-\vec{\tau} \cdot \vec{\Omega} \mathcal{Q}_{A}^{2} \hat{t} \psi(i \not \partial)^{-1} \psi\right]_{5} \beta\right| \omega, \alpha\right\rangle f_{\alpha}^{-}(\omega) \\
& +\sum_{\beta}\langle\alpha|\vec{\tau} \cdot \vec{\Omega}| \beta\rangle\left[\left\langle\omega, \beta\left|\mathcal{Q}_{A}^{2} \beta \psi(i \not \partial)^{-1} \psi\right| \omega, \alpha\right\rangle g_{\alpha \beta}^{+}(\omega)\right. \\
& \left.\left.+\left\langle\omega, \beta\left|\mathcal{Q}_{A}^{2}\left[\psi(i \not \partial)^{-1} \psi\right]_{5} \beta\right| \omega, \alpha\right\rangle g_{\alpha \beta}^{-}(\omega)\right]\right\} .
\end{aligned}
$$

In addition to the spectral functions for the linear matrix elements (5.10) we have introduced analogous objects for the bi-linear matrix elements

$$
g_{\alpha \beta}^{ \pm}(\omega)=\sum_{i=0}^{2} c_{i} \frac{\left(\omega \pm \epsilon_{\alpha}\right)\left(\omega \pm \epsilon_{\beta}\right)+\Lambda_{i}^{2}}{\left(\omega^{2}-\epsilon_{\alpha}^{2}-\Lambda_{i}^{2}+i \epsilon\right)\left(\omega^{2}-\epsilon_{\beta}^{2}-\Lambda_{i}^{2}+i \epsilon\right)} \pm \frac{\left(\omega \pm \epsilon_{\alpha}\right)\left(\omega \pm \epsilon_{\beta}\right)}{\left(\omega^{2}-\epsilon_{\alpha}^{2}+i \epsilon\right)\left(\omega^{2}-\epsilon_{\beta}^{2}+i \epsilon\right)} .
$$

The first two terms in eq (D.1), which do not contain the angular velocity $\vec{\Omega}$, have already been discussed in section 5 . The terms, which contain the time coordinate operator $\hat{t}$, originate from the time dependence of the collective coordinates (5.5). Finally the bi-linear pieces stem from expanding $\mathbf{D}^{(\pi)}$ and $\mathbf{D}_{5}^{(\pi)}$ to linear order in $\vec{\Omega}$, cf. eq (2.2). In eq (D.1) the abbreviation $\mathcal{Q}_{A}$ refers to the collectively rotated charge matrix at a distinct point in time. The same is true for the angular velocity. To be specific

$$
\mathcal{Q}_{A}=A^{\dagger}(0) \mathcal{Q} A(0) \quad \text { and } \quad \frac{i}{2} \vec{\tau} \cdot \vec{\Omega}=\left.A^{\dagger}(t) \frac{d}{d t} A(t)\right|_{t=0},
$$

as the time-dependence of the collective coordinates has been treated according to (5.5).

Before further manipulating (D.1) it is illuminating to discuss the relation to the sum rules at sub-leading order. The Adler sum rule for (anti) neutrino nucleon scattering involves the isospin operator. The constant of proportionality which relates this operator to the collectively rotated angular velocities is the moment of inertia, $\alpha^{2}$, cf. eq (2.20). The latter is obtained by expanding the regularized action to quadratic order in $\vec{\Omega}$,

$$
\begin{aligned}
\left.\frac{\partial^{2} \mathcal{A}}{\partial \Omega_{l} \partial \Omega_{m}}\right|_{\vec{\Omega}=0} & =\frac{i}{2} N_{C} \sum_{i=0}^{2} c_{i} \int \frac{d \omega}{2 \pi} \sum_{\alpha \beta}\left\langle\alpha\left|\tau_{l}\right| \beta\right\rangle\left\langle\beta\left|\tau_{m}\right| \alpha\right\rangle \frac{\omega^{2}+\epsilon_{\alpha} \epsilon_{\beta}+\Lambda_{i}^{2}}{\left(\omega^{2}-\epsilon_{\alpha}^{2}-\Lambda_{i}^{2}+i \epsilon\right)\left(\omega^{2}-\epsilon_{\beta}^{2}-\Lambda_{i}^{2}+i \epsilon\right)} \\
& =\frac{i}{4} N_{C} \int \frac{d \omega}{2 \pi} \sum_{\alpha \beta}\left\langle\alpha\left|\tau_{l}\right| \beta\right\rangle\left\langle\beta\left|\tau_{m}\right| \alpha\right\rangle\left[g_{\alpha \beta}^{+}(\omega)+g_{\alpha \beta}^{-}(\omega)\right]
\end{aligned}
$$

\footnotetext{
${ }^{1}$ This rotation is inherited by the iso-vector-vector piece in the flavor matrix which is analogous to $\mathcal{Q}_{A}$.
} 
In the second step we have used the property that the spectral integrals over those pieces which are odd in $\omega$ vanish. Although in practice this Cauchy integral could easily be performed, the obvious relation to the sum of the spectral functions, $g^{+}+g^{-}$, shows that the Adler sum rule will be satisfied. The reason is that in this channel the integration over $x$ will lead to the replacement $\psi(i \not \partial)^{-1} \psi \rightarrow \beta$ after assuming the Bjorken limit. Similarly one might want to consider the axial singlet charge which receives a non-vanishing contribution only at subleading order in $1 / N_{C}$. The generating expression is obtained from eqs (6.1) and (6.2) by putting $T_{\mathrm{a}}^{a}=1$ and $a^{\mu}=(0,0,0,1)$ while all vector quantities are set to zero. This yields

$$
\begin{array}{r}
-i N_{C} \Omega_{3} \int \frac{d \omega}{2 \pi} \sum_{\alpha \beta}\left\langle\alpha\left|\tau_{3}\right| \beta\right\rangle\left\langle\beta\left|\alpha_{3} \gamma_{5}\right| \alpha\right\rangle \frac{\omega^{2}+\epsilon_{\alpha} \epsilon_{\beta}}{\left(\omega^{2}-\epsilon_{\alpha}^{2}+i \epsilon\right)\left(\omega^{2}-\epsilon_{\beta}^{2}+i \epsilon\right)} \\
=i N_{C} \Omega_{3} \int \frac{d \omega}{2 \pi} \sum_{\alpha \beta}\left\langle\alpha\left|\tau_{3}\right| \beta\right\rangle\left\langle\beta\left|\alpha_{3} \gamma_{5}\right| \alpha\right\rangle\left[g_{\alpha \beta}^{+}(\omega)-g_{\alpha \beta}^{-}(\omega)\right] .
\end{array}
$$

As was to be expected the dependence on the cut-off, $\Lambda_{i}$ dropped out because this quantity is associated to the imaginary part of the action. However, it is remarkable that the difference $g^{+}-g^{-}$of the spectral functions appeared rather than their sum. On the level of the hadronic tensor a difference between the last and next-to-last terms in (D.1) can only be gained by the prescription (4.7) since the generator for the axial singlet charge $\left(\gamma_{3} \gamma_{5}\right)$ commutes with the Dirac matrix $\beta$. Of course, in view of the previous discussions this result was to be expected.

We now continue to compute the hadronic tensor by varying (D.1) with respect to the vector sources $v^{\mu}\left(\xi_{1}\right)$ and $v^{\nu}\left(\xi_{2}\right)$. At first sight, this would yield contributions from the forward and backward moving quarks for each single term in (D.1). However, this is not the case for those terms which contain the time-coordinate operator $\hat{t}$ because it may act on a state like $|\vec{\xi}, t=0\rangle$ which in turn gives zero contribution. In what follows we will concentrate on the treatment of that operator. At most it will contribute a factor linear in the time-coordinate which is integrated over when calculation the Compton tensor (1.7). For that calculation the coordinate $\xi=t$ can be expressed as a derivative with respect to $q^{0}$. In turn this derivative can be re-written as a derivative with respect to Bjorken $x$. In the nucleon rest frame we have $\partial x / \partial q^{0}=-1 / 2 M_{N}$. Once this is established, the calculation of the hadronic tensor proceeds as in section 5 and yields an expression analogous to eq (5.15). Differentiating with respect to $x$ then simply gives a factor $\lambda M_{N}$. In its full glory the hadronic tensor finally reads

$$
\begin{aligned}
& W_{\mu \nu} \stackrel{\mathrm{Bj}}{\longrightarrow}-i M_{N} \frac{N_{C}}{4} \int \frac{d \omega}{2 \pi} \sum_{\alpha} \int d^{3} \xi \int \frac{d \lambda}{2 \pi} \mathrm{e}^{i M_{N} x \lambda}\langle N| \\
& \left\{\left.\left[\bar{\Psi}_{\alpha}(\vec{\xi}) \mathcal{Q}_{A}^{2} \gamma_{\mu} \not h \gamma_{\nu} \Psi_{\alpha}\left(\vec{\xi}+\lambda \hat{e}_{3}\right) \mathrm{e}^{-i \lambda \omega}-\bar{\Psi}_{\alpha}(\vec{\xi}) \mathcal{Q}_{A}^{2} \gamma_{\nu} \not h \gamma_{\mu} \Psi_{\alpha}\left(\vec{\xi}-\lambda \hat{e}_{3}\right) \mathrm{e}^{i \lambda \omega}\right] f_{\alpha}^{+}(\omega)\right|_{\mathrm{p}}\right. \\
& +\left.\left[\bar{\Psi}_{\alpha}(\vec{\xi}) \mathcal{Q}_{A}^{2}\left(\gamma_{\mu} \not h \gamma_{\nu}\right)_{5} \Psi_{\alpha}\left(\vec{\xi}-\lambda \hat{e}_{3}\right) \mathrm{e}^{-i \lambda \omega}-\bar{\Psi}_{\alpha}(\vec{\xi}) \mathcal{Q}_{A}^{2}\left(\gamma_{\nu} \not h \gamma_{\mu}\right)_{5} \Psi_{\alpha}\left(\vec{\xi}+\lambda \hat{e}_{3}\right) \mathrm{e}^{i \lambda \omega}\right] f_{\alpha}^{-}(\omega)\right|_{\mathrm{p}} \\
& +\left.\frac{i \lambda}{4}\left[\bar{\Psi}_{\alpha}(\vec{\xi}) \vec{\tau} \cdot \vec{\Omega} \mathcal{Q}_{A}^{2} \gamma_{\mu} \not h \gamma_{\nu} \Psi_{\alpha}\left(\vec{\xi}+\lambda \hat{e}_{3}\right) \mathrm{e}^{-i \lambda \omega}+\bar{\Psi}_{\alpha}(\vec{\xi}) \mathcal{Q}_{A}^{2} \vec{\tau} \cdot \vec{\Omega} \gamma_{\nu} \not h \gamma_{\mu} \Psi_{\alpha}\left(\vec{\xi}-\lambda \hat{e}_{3}\right) \mathrm{e}^{i \lambda \omega}\right] f_{\alpha}^{+}(\omega)\right|_{\mathrm{p}} \\
& +\left.\frac{i \lambda}{4}\left[\bar{\Psi}_{\alpha}(\vec{\xi}) \vec{\tau} \cdot \vec{\Omega} \mathcal{Q}_{A}^{2}\left(\gamma_{\mu} \not h \gamma_{\nu}\right)_{5} \Psi_{\alpha}\left(\vec{\xi}-\lambda \hat{e}_{3}\right) \mathrm{e}^{-i \lambda \omega}+\bar{\Psi}_{\alpha}(\vec{\xi}) \mathcal{Q}_{A}^{2} \vec{\tau} \cdot \vec{\Omega}\left(\gamma_{\nu} \not h \gamma_{\mu}\right)_{5} \Psi_{\alpha}\left(\vec{\xi}+\lambda \hat{e}_{3}\right) \mathrm{e}^{i \lambda \omega}\right] f_{\alpha}^{-}(\omega)\right|_{\mathrm{p}}
\end{aligned}
$$




$$
\begin{aligned}
& +\sum_{\beta}\langle\alpha|\vec{\tau} \cdot \vec{\Omega}| \beta\rangle\left(\left.\left[\bar{\Psi}_{\beta}(\vec{\xi}) \mathcal{Q}_{A}^{2} \gamma_{\mu} \not h \gamma_{\nu} \Psi_{\alpha}\left(\vec{\xi}+\lambda \hat{e}_{3}\right) \mathrm{e}^{-i \lambda \omega}-\bar{\Psi}_{\beta}(\vec{\xi}) \mathcal{Q}_{A}^{2} \gamma_{\nu} \not h \gamma_{\mu} \Psi_{\alpha}\left(\vec{\xi}-\lambda \hat{e}_{3}\right) \mathrm{e}^{i \lambda \omega}\right] g_{\alpha \beta}^{+}(\omega)\right|_{\mathrm{p}}\right. \\
& \left.\left.+\left.\left[\bar{\Psi}_{\beta}(\vec{\xi}) \mathcal{Q}_{A}^{2}\left(\gamma_{\mu} \not h \gamma_{\nu}\right)_{5} \Psi_{\alpha}\left(\vec{\xi}-\lambda \hat{e}_{3}\right) \mathrm{e}^{-i \lambda \omega}-\bar{\Psi}_{\beta}(\vec{\xi}) \mathcal{Q}_{A}^{2}\left(\gamma_{\nu} \not h \gamma_{\mu}\right)_{5} \Psi_{\alpha}\left(\vec{\xi}+\lambda \hat{e}_{3}\right) \mathrm{e}^{i \lambda \omega}\right] g_{\alpha \beta}^{-}(\omega)\right|_{\mathrm{p}}\right)\right\}|N\rangle .
\end{aligned}
$$

Here have made use of the fact that $\vec{\tau} \cdot \vec{\Omega}$ does not change the parity of single quark states. This implies that in the double sum the states $|\alpha\rangle$ and $|\beta\rangle$ carry the same parity quantum number. In turn this allowed us to simply compensate factors of the Dirac matrix $\beta$ by spatial reflections. In the double sum the pole contribution is obtained by summing over all possible frequencies which lead to singularities in the spectral functions (D.2). This summation is indicated in figure D.1.

Figure D.1: Summing the poles of the bi-local cranking corrections. The angular velocity $\vec{\Omega}$ acts as a perturbation to the quark propagator in the soliton background.

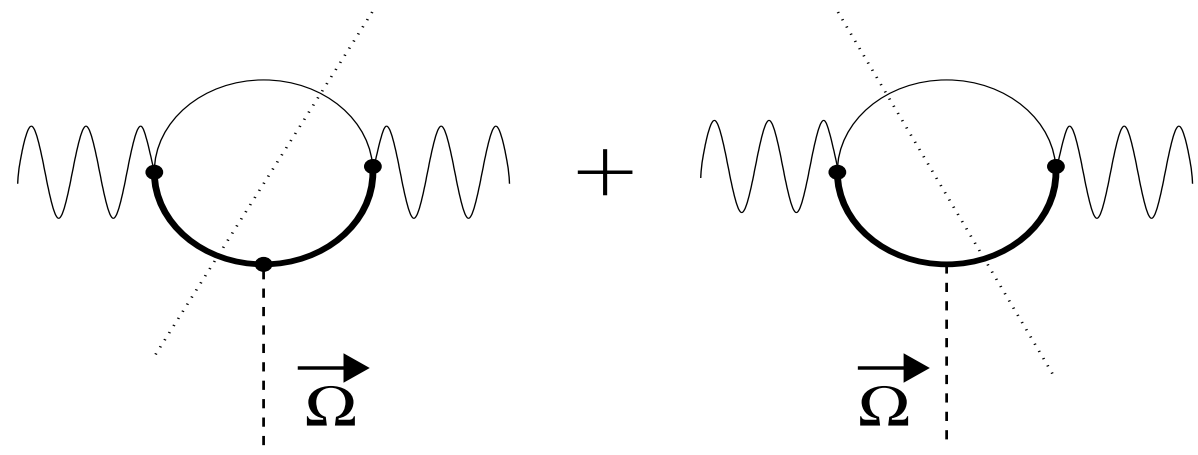

At this point it is worth to briefly comment on the possible occurance of double poles, i.e. $\epsilon_{\alpha}^{2}=\epsilon_{\beta}^{2}+\Lambda_{i}^{2}$ and $\epsilon_{\alpha}^{2}=\epsilon_{\beta}^{2}$ in the regularized and un-regularized cases, respectively. According to Cutkosky's rules they contribute derivatives of $\delta$-functions to the absorptive part of the amplitude: $-2 \pi i \partial \delta\left(\omega^{2}-\epsilon_{\alpha}^{2}\right) / \partial \omega^{2}$. This is directly linked to the double poles which correspondingly appear in the spectral integrals for the charges (D.5) and the moment of inertia (D.4). They can as well be expressed as derivatives of single poles. In this way the sum rules are ensured to be satisfied.

A priori the product $\vec{\tau} \cdot \vec{\Omega} \mathcal{Q}_{A}^{2}$ is plagued by ordering ambiguities when imposing the quantization rules (2.20) for the collective coordinates. To guarantee hermiticity we assume the symmetric ordering. That is

$$
\frac{1}{2}\left\{J_{i}, \mathcal{Q}_{A}^{2}\right\}=\frac{1}{18}\left(5 J_{i}-I_{3} \tau_{i}\right)
$$

where we have also used that the matrix elements are evaluated in the subspace of nucleon states. This allowed us to employ eq (2.21). In particular, the symmetric ordering permitted us to identify $\vec{\tau} \cdot \vec{\Omega} \mathcal{Q}_{A}^{2}=\mathcal{Q}_{A}^{2} \vec{\tau} \cdot \vec{\Omega}$. For notational brevity we will maintain that expression in what follows, keeping in mind that it should be substituted by (D.7). Contracting the hadronic tensor 
(D.6) with the appropriate projector from table 5.1 gives the unpolarized structure function to sub-leading order in $1 / N_{C}$, see also eq (5.16),

$$
\begin{gathered}
f_{1}(x)=-i M_{N} \frac{N_{C}}{2} \int \frac{d \omega}{2 \pi} \sum_{\alpha} \int d^{3} \xi \int \frac{d \lambda}{2 \pi} \mathrm{e}^{i M_{N} x \lambda}\langle N| \\
\times\left\{\left[\bar{\Psi}_{\alpha}(\vec{\xi}) \mathcal{Q}_{A}^{2} \not h \Psi_{\alpha}\left(\vec{\xi}+\lambda \hat{e}_{3}\right) \mathrm{e}^{-i \omega \lambda}-\bar{\Psi}_{\alpha}(\vec{\xi}) \mathcal{Q}_{A}^{2} \not h \Psi_{\alpha}\left(\vec{\xi}-\lambda \hat{e}_{3}\right)\right) \mathrm{e}^{i \omega \lambda}\right]\left(\sum_{i=0}^{2} c_{i} \frac{\omega+\epsilon_{\alpha}}{\omega^{2}-\epsilon_{\alpha}^{2}-\Lambda_{i}^{2}+i \epsilon}\right)_{\mathrm{p}} \\
\left.+\frac{i \lambda}{4}\left[\bar{\Psi}_{\alpha}(\vec{\xi}) \vec{\tau} \cdot \vec{\Omega} \mathcal{Q}_{A}^{2} \not h \Psi_{\alpha}\left(\vec{\xi}+\lambda \hat{e}_{3}\right) \mathrm{e}^{-i \omega \lambda}+\bar{\Psi}_{\alpha}(\vec{\xi}) \vec{\tau} \cdot \vec{\Omega} \mathcal{Q}_{A}^{2} \not h \Psi_{\alpha}\left(\vec{\xi}-\lambda \hat{e}_{3}\right)\right) \mathrm{e}^{i \omega \lambda}\right]\left(\frac{\omega+\epsilon_{\alpha}}{\omega^{2}-\epsilon_{\alpha}^{2}+i \epsilon}\right)_{\mathrm{p}} \\
\left.+\sum_{\beta}\left[\bar{\Psi}_{\beta}(\vec{\xi}) \mathcal{Q}_{A}^{2} \not h \Psi_{\alpha}\left(\vec{\xi}+\lambda \hat{e}_{3}\right) \mathrm{e}^{-i \omega \lambda}-\bar{\Psi}_{\beta}(\vec{\xi}) \mathcal{Q}_{A}^{2} \not h \Psi_{\alpha}\left(\vec{\xi}-\lambda \hat{e}_{3}\right)\right) \mathrm{e}^{i \omega \lambda}\right] \\
\left.\times\langle\alpha|\vec{\tau} \cdot \vec{\Omega}| \beta\rangle\left(\frac{\left(\omega+\epsilon_{\alpha}\right)\left(\omega+\epsilon_{\beta}\right)}{\left(\omega^{2}-\epsilon_{\alpha}^{2}+i \epsilon\right)\left(\omega^{2}-\epsilon_{\beta}^{2}+i \epsilon\right)}\right)_{\mathrm{p}}\right\}|N\rangle .
\end{gathered}
$$

We observe that in the sub-leading contributions the dependence on the cut-off, $\Lambda_{i}$ has disappeared as the remaining spectral functions are associated with

$$
\begin{aligned}
f_{\alpha}^{+}(\omega)+f_{\alpha}^{-}(-\omega) & =2 \frac{\omega+\epsilon_{\alpha}}{\omega^{2}-\epsilon_{\alpha}^{2}+i \epsilon} \\
g_{\alpha \beta}^{+}(\omega)-g_{\alpha \beta}^{-}(-\omega) & =2 \frac{\left(\omega+\epsilon_{\alpha}\right)\left(\omega+\epsilon_{\beta}\right)}{\left(\omega^{2}-\epsilon_{\alpha}^{2}+i \epsilon\right)\left(\omega^{2}-\epsilon_{\beta}^{2}+i \epsilon\right)} .
\end{aligned}
$$

Actually this finding shows that previous computations [13, 14] regularizing the vacuum contribution to the Gottfried sum rule are not consistent with the present analysis of the Compton amplitude. This has to be contrasted with the iso-vector part of the structure function for the neutrino nucleon scattering. The only significant difference is the opposite sign for the terms involving the crossed ordering $(\nu \mu)$, i.e. backward propagating quarks. The reason is that neutrino scattering involves the exchange of a charged gauge boson. Hence the iso-vector projection switches sign when going from the product of currents $J_{\mu}(\xi) J_{\nu}^{\dagger}(0)$ to the hermitian conjugate $J_{\nu}(0) J_{\mu}^{\dagger}(\xi)$. Having noted that, we find the spectral function to be

$$
g_{\alpha \beta}^{+}(\omega)+g_{\alpha \beta}^{-}(-\omega)=2 \sum_{i=0}^{2} c_{i} \frac{\left(\omega+\epsilon_{\alpha}\right)\left(\omega+\epsilon_{\beta}\right)}{\left(\omega^{2}-\epsilon_{\alpha}^{2}-\Lambda_{i}^{2}+i \epsilon\right)\left(\omega^{2}-\epsilon_{\beta}^{2}-\Lambda_{i}^{2}+i \epsilon\right)}
$$

and hence the Adler sum rule to be satisfied, $c f$. eq (D.4).

We can now calculate the appropriate matrix elements by again using the grand-spin and parity properties of the single quark states,

$$
\begin{aligned}
& f_{1}(x)=\text { eq }(5.16)+i I_{3} \frac{M_{N} N_{C}}{36 \alpha^{2}} \int \frac{d \omega}{2 \pi} \sum_{\alpha} \int \frac{d \lambda}{2 \pi} \mathrm{e}^{i M_{N} x \lambda} \int d^{3} \xi \\
& \times\left\{\frac{3 i \lambda}{4}\left[\Psi_{\alpha}^{\dagger}(\vec{\xi})\left(1-\alpha_{3}\right) \Psi_{\alpha}\left(\vec{\xi}+\lambda \hat{e}_{3}\right) \mathrm{e}^{-i \omega \lambda}+\Psi_{\alpha}^{\dagger}(\vec{\xi})\left(1-\alpha_{3}\right) \Psi_{\alpha}\left(\vec{\xi}-\lambda \hat{e}_{3}\right) \mathrm{e}^{i \omega \lambda}\right]\left(\frac{\omega+\epsilon_{\alpha}}{\omega^{2}-\epsilon_{\alpha}^{2}+i \epsilon}\right)_{\mathrm{p}}\right. \\
& \quad+\sum_{\beta}\left(\frac{\left(\omega+\epsilon_{\alpha}\right)\left(\omega+\epsilon_{\beta}\right)}{\left(\omega^{2}-\epsilon_{\alpha}^{2}+i \epsilon\right)\left(\omega^{2}-\epsilon_{\beta}^{2}+i \epsilon\right)}\right)_{\mathrm{p}}
\end{aligned}
$$




$$
\begin{aligned}
& \times\left(4\left\langle\alpha\left|\tau_{1}\right| \beta\right\rangle\left[\Psi_{\alpha}^{\dagger}(\vec{\xi}) \tau_{1}\left(1-\alpha_{3}\right) \Psi_{\alpha}\left(\vec{\xi}+\lambda \hat{e}_{3}\right) \mathrm{e}^{-i \omega \lambda}-\Psi_{\alpha}^{\dagger}(\vec{\xi}) \tau_{1}\left(1-\alpha_{3}\right) \Psi_{\alpha}\left(\vec{\xi}-\lambda \hat{e}_{3}\right) \mathrm{e}^{i \omega \lambda}\right]\right. \\
& \left.\left.\quad-\left\langle\alpha\left|\tau_{3}\right| \beta\right\rangle\left[\Psi_{\alpha}^{\dagger}(\vec{\xi}) \tau_{3}\left(1-\alpha_{3}\right) \Psi_{\alpha}\left(\vec{\xi}+\lambda \hat{e}_{3}\right) \mathrm{e}^{-i \omega \lambda}-\Psi_{\alpha}^{\dagger}(\vec{\xi}) \tau_{3}\left(1-\alpha_{3}\right) \Psi_{\alpha}\left(\vec{\xi}-\lambda \hat{e}_{3}\right) \mathrm{e}^{i \omega \lambda}\right]\right)\right\}
\end{aligned}
$$

The appearance of two terms in the cranking piece reflects the fact that only an axial symmetry is left after selecting a direction for the photon momentum. Also note that (D.12) does not contain the nucleon spin operator. Of course, this is expected for an unpolarized structure function.

The anti-symmetric piece of the hadronic tensor reads

$$
\begin{aligned}
& W_{\mu \nu}^{\mathrm{A}}=-M_{N} \frac{N_{C}}{2} \epsilon_{\mu \nu \rho \sigma} \mathrm{n}^{\rho} \int \frac{d \omega}{2 \pi} \sum_{\alpha} \int d^{3} \xi \int \frac{d \lambda}{2 \pi} \mathrm{e}^{i M_{N} x \lambda}\langle N| \\
& \times\left\{\left[\bar{\Psi}_{\alpha}(\vec{\xi}) \mathcal{Q}_{A}^{2} \gamma^{\sigma} \gamma_{5} \Psi_{\alpha}\left(\vec{\xi}+\lambda \hat{e}_{3}\right) \mathrm{e}^{-i \omega \lambda}\right.\right. \\
& \left.\left.\quad+\bar{\Psi}_{\alpha}(\vec{\xi}) \mathcal{Q}_{A}^{2} \gamma^{\sigma} \gamma_{5} \Psi_{\alpha}\left(\vec{\xi}-\lambda \hat{e}_{3}\right)\right) \mathrm{e}^{i \omega \lambda}\right]\left(\sum_{i=0}^{2} c_{i} \frac{\omega+\epsilon_{\alpha}}{\omega^{2}-\epsilon_{\alpha}^{2}-\Lambda_{i}^{2}+i \epsilon}\right)_{\mathrm{p}} \\
& +\frac{i \lambda}{4}\left[\bar{\Psi}_{\alpha}(\vec{\xi}) \vec{\tau} \cdot \vec{\Omega} \mathcal{Q}_{A}^{2} \gamma^{\sigma} \gamma_{5} \Psi_{\alpha}\left(\vec{\xi}+\lambda \hat{e}_{3}\right) \mathrm{e}^{-i \omega \lambda}\right. \\
& \left.\left.\quad-\bar{\Psi}_{\alpha}(\vec{\xi}) \vec{\tau} \cdot \vec{\Omega} \mathcal{Q}_{A}^{2} \gamma^{\sigma} \gamma_{5} \Psi_{\alpha}\left(\vec{\xi}-\lambda \hat{e}_{3}\right)\right) \mathrm{e}^{i \omega \lambda}\right]\left(\frac{\omega+\epsilon_{\alpha}}{\omega^{2}-\epsilon_{\alpha}^{2}+i \epsilon}\right)_{\mathrm{p}} \\
& \left.+\sum_{\beta}\left[\bar{\Psi}_{\beta}(\vec{\xi}) \mathcal{Q}_{A}^{2} \gamma^{\sigma} \gamma_{5} \Psi_{\alpha}\left(\vec{\xi}+\lambda \hat{e}_{3}\right) \mathrm{e}^{-i \omega \lambda}+\bar{\Psi}_{\beta}(\vec{\xi}) \mathcal{Q}_{A}^{2} \gamma^{\sigma} \gamma_{5} \Psi_{\alpha}\left(\vec{\xi}-\lambda \hat{e}_{3}\right)\right) \mathrm{e}^{i \omega \lambda}\right] \\
& \left.\times\langle\alpha|\vec{\tau} \cdot \vec{\Omega}| \beta\rangle\left(\frac{\left(\omega+\epsilon_{\alpha}\right)\left(\omega+\epsilon_{\beta}\right)}{\left(\omega^{2}-\epsilon_{\alpha}^{2}+i \epsilon\right)\left(\omega^{2}-\epsilon_{\beta}^{2}+i \epsilon\right)}\right)_{\mathrm{p}}\right\}|N\rangle .
\end{aligned}
$$

We note that after contracting the cranking corrections with the pertinent projectors from table 5.1, the parity properties of the single quark states enforce the combination of the Dirac matrices and the gradient expansion in $\lambda$ to be odd under grand-spin reflection $\left(\gamma_{5}\right.$ is even under this operation). Hence the isospin part must be odd as well. Therefore the cranking corrections to the polarized structure functions are iso-singlets in the space of the collective coordinates. To be specific,

$$
\begin{aligned}
g_{1}(x)= & \text { eq }(5.18)+\frac{5 i}{6 \alpha^{2}} M_{N} N_{C} \int \frac{d \omega}{2 \pi} \sum_{\alpha} \int \frac{d \lambda}{2 \pi} \mathrm{e}^{i M_{N} x \lambda} \int d^{3} \xi \\
& \times \frac{i \lambda}{4}\left[\Psi_{\alpha}^{\dagger}(\vec{\xi}) \tau_{3}\left(1-\alpha_{3}\right) \gamma_{5} \Psi_{\alpha}\left(\vec{\xi}+\lambda \hat{e}_{3}\right) \mathrm{e}^{-i \omega \lambda}\right. \\
& \left.-\Psi_{\alpha}^{\dagger}(\vec{\xi}) \tau_{3}\left(1-\alpha_{3}\right) \gamma_{5} \Psi_{\alpha}\left(\vec{\xi}-\lambda \hat{e}_{3}\right) \mathrm{e}^{i \omega \lambda}\right]\left(\frac{\omega+\epsilon_{\alpha}}{\omega^{2}-\epsilon_{\alpha}^{2}+i \epsilon}\right)_{\mathrm{p}} \\
+ & \sum_{\beta}\left(\frac{\left(\omega+\epsilon_{\alpha}\right)\left(\omega+\epsilon_{\beta}\right)}{\left(\omega^{2}-\epsilon_{\alpha}^{2}+i \epsilon\right)\left(\omega^{2}-\epsilon_{\beta}^{2}+i \epsilon\right)}\right)_{\mathrm{p}}^{\left\langle\alpha\left|\tau_{3}\right| \beta\right\rangle} \\
& \left.\times\left[\Psi_{\alpha}^{\dagger}(\vec{\xi})\left(1-\alpha_{3}\right) \gamma_{5} \Psi_{\alpha}\left(\vec{\xi}+\lambda \hat{e}_{3}\right) \mathrm{e}^{-i \omega \lambda}+\Psi_{\alpha}^{\dagger}(\vec{\xi})\left(1-\alpha_{3}\right) \gamma_{5} \Psi_{\alpha}\left(\vec{\xi}-\lambda \hat{e}_{3}\right) \mathrm{e}^{i \omega \lambda}\right]\right\}
\end{aligned}
$$


and

$$
\begin{aligned}
g_{T}(x)= & \text { eq }(5.19)+\frac{5 i}{6 \alpha^{2}} M_{N} N_{C} \int \frac{d \omega}{2 \pi} \sum_{\alpha} \int \frac{d \lambda}{2 \pi} \mathrm{e}^{i M_{N} x \lambda} \int d^{3} \xi \\
\times & =\frac{i \lambda}{4}\left[\Psi_{\alpha}^{\dagger}(\vec{\xi}) \tau_{1} \alpha_{1} \gamma_{5} \Psi_{\alpha}\left(\vec{\xi}+\lambda \hat{e}_{3}\right) \mathrm{e}^{-i \omega \lambda}\right. \\
& \left.-\Psi_{\alpha}^{\dagger}(\vec{\xi}) \tau_{1} \alpha_{1} \gamma_{5} \Psi_{\alpha}\left(\vec{\xi}-\lambda \hat{e}_{3}\right) \mathrm{e}^{i \omega \lambda}\right]\left(\frac{\omega+\epsilon_{\alpha}}{\omega^{2}-\epsilon_{\alpha}^{2}+i \epsilon}\right)_{\mathrm{p}} \\
+ & \sum_{\beta}\left(\frac{\left(\omega+\epsilon_{\alpha}\right)\left(\omega+\epsilon_{\beta}\right)}{\left(\omega^{2}-\epsilon_{\alpha}^{2}+i \epsilon\right)\left(\omega^{2}-\epsilon_{\beta}^{2}+i \epsilon\right)}\right)_{\mathrm{p}}^{\left\langle\alpha\left|\tau_{3}\right| \beta\right\rangle} \\
& \left.\times\left[\Psi_{\alpha}^{\dagger}(\vec{\xi}) \gamma_{1} \Psi_{\alpha}\left(\vec{\xi}+\lambda \hat{e}_{3}\right) \mathrm{e}^{-i \omega \lambda}+\Psi_{\alpha}^{\dagger}(\vec{\xi}) \gamma_{1} \Psi_{\alpha}\left(\vec{\xi}-\lambda \hat{e}_{3}\right) \mathrm{e}^{i \omega \lambda}\right]\right\},
\end{aligned}
$$

after taking the collective coordinate (2.20) and (2.21). As it is the case for static properties we find that only either the iso-scalar or iso-vector contribution to any structure function become regularized but not both. For the polarized structure function the result that only the isovector part is regularized was anticipated because we know how to relate their zeroth moments to nucleon charges. In case of the unpolarized structure function it comes as a surprise that the iso-scalar part rather than the iso-vector piece undergoes regularization. However for this structure function we do not have a normalized sum rule at hand. As discussed, for unpolarized structure function which enters the Adler sum rule the situation is opposite.

\section{References}

[1] R. G. Roberts, The Structure of the Proton, Cambridge Monographs on Mathematical Physics, 1990.

[2] M. Glück, E. Reya and A. Vogt, Z. Phys. C67 (1995) 433.

M. Glück, E. Reya M. Stratmann and M. Vogelsang, Phys. Rev. D53 (1996) 4775.

M. Vogelsang, Phys. Rev. D54 (1996) 2023.

A. D. Martin, R.G. Roberts, W.J. Stirling and R.S. Thorne, Eur. Phys. J. C4 (1998) 463.

E. Ruiz Arriola, Nucl. Phys. A641 (1998) 461,

M. Glück, E. Reya and A. Vogt, Eur. J. Phys. C5 (1998) 461,

H. L. Lai et al., Global Analysis of Parton Structure of the Nucleon: CTEQ5 Parton Distributions, hep-ph/9903282.

[3] R. L. Jaffe, Phys. Rev. D11 (1975) 1953. 
[4] C. J. Benesh and G. A. Miller, Phys. Rev. D36 (1987) 1344, A. I. Signal and A. W. Thomas, Phys. Lett. B211 (1988) 481, A. W. Schreiber, A. I. Signal and A. W. Thomas, Phys. Rev. D44 (1991) 2653, A. W. Schreiber, P. J. Mulders, A. I. Signal and A. W. Thomas, Phys. Rev. D45 (1992) 3069, X. I. Song and J. S. McCarthy, Phys. Rev. D49 (1994) 3169,

X. I. Song, Phys. Rev. D55 (1996) 1955.

V. Sanjose and V. Vento, Phys. Lett. B225 (1988) 15, Nucl. Phys. A501 (1989) 481,

M. Traini, V. Vento, A. Mair and A. Zambarda, Nucl. Phys. A614 (1997) 472.

H. Holtmann, A. Szczurek and J. Speth, Nucl. Phys. A596 (1996) 631.

H. Meyer and P. J. Mulders, Nucl. Phys. A528 (1991) 589,

S. A. Kulagin, W. Melnitchouck, T. Weigl and W. Weise, Nucl. Phys. A597 (1996) 515.

[5] For reviews on Skyrme type soliton models see:

G. Holzwarth and B. Schwesinger, Rep. Prog. Phys. 49 (1986) 825.

I. Zahed and G. E. Brown, Phys. Rep. 142 (1986) 481.

Ulf-G. Meißner, Phys. Rep. 161 (1988) 213.

B. Schwesinger, H. Weigel, G. Holzwarth and A. Hayashi, Phys. Rep. 173 (1989) 173.

H. Weigel, Int. J. Mod. Phys. A11 (1996) 2419.

[6] Y. Nambu and G. Jona-Lasinio, Phys. Rev. 122 (1961) 345; 124 (1961) 246.

[7] D. Ebert and H. Reinhardt, Nucl. Phys. B271 (1986) 188.

[8] U. Vogl and W. Weise, Prog. Part. Nucl. Phys. 27 (1991) 195.

[9] R. Alkofer, H. Reinhardt and H. Weigel, Phys. Rep. 265 (1996) 139.

C. V. Christov et al., Prog. Part. Nucl. Phys. 37 (1996) 91.

[10] H. Weigel, L. Gamberg and H. Reinhardt, Mod. Phys. Lett. A11 (1996) 3021, Phys. Lett. B399 (1997) 287,

O. Schröder, H. Reinhardt and H. Weigel, Phys. Lett. B439 (1998) 398.

[11] H. Weigel, L. Gamberg and H. Reinhardt, Phys. Rev. D55 (1997) 6910.

L. Gamberg, H. Reinhardt and H. Weigel, Phys. Rev. D58 (1998) 054014.

[12] D. I. Diakonov et al., Nucl. Phys. B480 (1996) 341, Phys. Rev. D56 (1997) 4069.

[13] M. Wakamatsu and T. Kubota, Phys. Rev. D57 (1998) 5755.

[14] P. V. Pobylitsa et al., Phys. Rev. D59 (1999) 034024.

[15] K. Tanikawa and S. Saito, Nucleon Structure functions in the chiral quark soliton model, Nagoya University preprint-DPNU-96-37.

[16] G. S. Adkins, C. R. Nappi and E. Witten, Nucl. Phys. B228 (1983) 552.

[17] R. M. Davidson and E. Ruiz Arriola, Phys. Lett. B348 (1995) 163.

[18] H. Weigel, Nucleon structure functions in a chiral soliton model, hep-ph/9902390.

[19] R. M. Davidson and E. Ruiz Arriola, Phys. Lett. 359 (1995) 273.

[20] W. Broniowski, E. Nikolov, G. Ripka and K. Goeke, Z. Phys. A 354 (1996) 421. 
[21] E. Ruiz Arriola and L. L. Salcedo, Nucl. Phys. A590 (1995) 703.

[22] E. Ruiz Arriola, Phys. Lett. 253 (1991) 430.

[23] C. Schüren, E. Ruiz Arriola and K. Goeke, Nucl. Phys. A 547 (1992) 612.

[24] C. Schüren, F. Döring, E. Ruiz Arriola and K. Goeke, Nucl. Phys. A565 (1993) 687.

[25] A. Dhar, R. Shankar and S. R. Waida, Phys. Rev. D31 (1985) 3256.

[26] M. Wakamatsu, Phys. Rev. D54 (1996) 6459. M. Wakamatsu

[27] S. Kahana and G. Ripka, Nucl. Phys. A429 (1984) 462.

[28] H. Reinhardt, Nucl. Phys. A503 (1989) 825.

[29] F. Döring et al., Nucl. Phys. A536 (1992) 548.

[30] H. Weigel, R. Alkofer and H. Reinhardt, Nucl. Phys. B387 (1992) 638.

[31] T. Frederico and G. A. Miller, Phys. Rev. D50 (1994) 210.

[32] J. L. Gervais, A. Jevicki and B. Sakita, Phys. Rep. 23 (1976) 281.

[33] M. Wakamatsu and T. Kubota, Chiral symmetry and the nucleon spin structure functions, hep$\mathrm{ph} / 9809443$.

O. Schröder, H. Reinhardt and H. Weigel, Nucleon structure functions in the three-flavor NJL model, hep-ph/9902322, Nucl. Phys. A, to be published.

[34] K. Gottfried, Phys. Rev. Lett. 18 (1967) 1174.

[35] R. L. Jaffe, Spin, Twist and Hadron Structure..., Erice Lectures 1995, hep-ph/9602236.

[36] R. L. Jaffe, Ann. Phys. (NY) 132 (1981) 32.

L. Gamberg, H. Reinhardt and H. Weigel, Int. J. Mod. Phys. A13 (1998) 5519.

[37] H. Burkhardt and W. N. Cottingham, Ann. Phys. (NY) 56 (1970) 453.

[38] H. Weigel, U. Zückert, R. Alkofer and H. Reinhardt, Nucl. Phys. A585 (1995) 513.

[39] F. Döring, C. Schüren, E. Ruiz Arriola, W. Watabe and K. Goeke, Nucl. Phys. A603 (1996) 415.

[40] K. Abe et al. (E143 Coll.), Phys. Rev. D58 (1998) 112003,

P. L. Anthony et al. (E155 Coll.), hep-ex/9901006.

[41] Th. Heinzl, Light Cone Dynamics of Particles and Fields, hep-ph/9812190.

[42] W. Bentz, T. Hama, T. Matsuki, K. Yazaki, NJL model on the light cone and pion structure function, hep-ph/9901377.

[43] F. E. Close, An Introduction to Quarks and Partons, Academic Press, 1979 and refs therein.

[44] M. Hillery, R. F. O’Conell, M. O. Scully and E. P. Wigner, Phys. Rep. 106 (1984) 121. 